\title{
Accidental SO(10) axion from gauged flavour
}

\author{
Luca Di Luzio \\ Deutsches Elektronen-Synchrotron (DESY), \\ Notkestraße 85, D-22607 Hamburg, Germany \\ E-mail: lukaluz@gmail.com
}

Abstract: An accidental U(1) Peccei-Quinn (PQ) symmetry automatically arises in a class of $\mathrm{SO}(10)$ unified theories upon gauging the $\mathrm{SU}(3)_{f}$ flavour group. The PQ symmetry is protected by the $\mathbb{Z}_{4} \times \mathbb{Z}_{3}$ center of $\mathrm{SO}(10) \times \mathrm{SU}(3)_{f}$ up to effective operators of canonical dimension six. However, high-scale contributions to the axion potential posing a PQ quality problem arise only at $d=9$. In the pre-inflationary $\mathrm{PQ}$ breaking scenario the axion mass window is predicted to be $m_{a} \in\left[7 \times 10^{-8}, 10^{-3}\right] \mathrm{eV}$, where the lower end is bounded by the seesaw scale and the upper end by iso-curvature fluctuations. A high-quality axion, that is immune to the PQ quality problem, is obtained for $m_{a} \gtrsim 0.02 \mathrm{eV}$. We finally offer a general perspective on the PQ quality problem in grand unified theories.

KEYwords: Beyond Standard Model, Global Symmetries, GUT

ARXIV EPRINT: 2008.09119 


\section{Contents}

1 Introduction 1

2 Gauging the way to the $\mathrm{SO}(10)$ axion 3

3 Peccei-Quinn quality 5

4 Axion's guts (physical axion field) $\quad 7$

4.1 A dominant SM-singlet and PQ-breaking VEV 8

4.2 Two SM-singlet and PQ-breaking VEVs 9

$\begin{array}{ll}4.3 \text { Axion domain wall problem? } & 10\end{array}$

$5 \quad \mathrm{SU}(3)_{f}$ sector $\quad 11$

$\begin{array}{lll}5.1 & \mathrm{SU}(3)_{f}^{3} \text { anomaly } & 11\end{array}$

$\begin{array}{lll}5.2 & \mathrm{SU}(3)_{f} \text { breaking } & 12\end{array}$

6 Axion phenomenology 13

$\begin{array}{lll}\text { 6.1 Pre-inflationary PQ breaking } & 13\end{array}$

$\begin{array}{lll}6.2 & \text { Post-inflationary PQ breaking } & 14\end{array}$

$\begin{array}{lll}7 & \text { Conclusions } & 16\end{array}$

$\begin{array}{ll}\text { A Low-energy axion couplings } & 17\end{array}$

\section{Introduction}

The Peccei-Quinn (PQ) $[1,2]$ mechanism relies on a global $\mathrm{U}(1)_{\mathrm{PQ}}$ symmetry that is broken in the infrared (IR) by the QCD anomaly. Global symmetries have no fundamental meaning, but they are rather understood to arise as accidental symmetries e.g. in quantum field theories. Well-known examples are baryon and lepton number in the Standard Model $(\mathrm{SM})$. Although an effective $\mathrm{U}(1)_{\mathrm{PQ}}$ is sometimes imposed "by hand", a proper $P Q$ theory should achieve that in an automatic way. This was the term used in the early days [3], when first attempts were put forth to get an accidental $\mathrm{U}(1)_{\mathrm{PQ}}$ in grand unified theories (GUTs). The quick decline of the electroweak Weinberg-Wilczek [4, 5] axion and the rise of the so-called invisible axion [6-9], brought in another related puzzle, known as the $P Q$ quality problem [3, 10-14]: why is $\mathrm{U}(1)_{\mathrm{PQ}}$ an extremely good symmetry of ultraviolet (UV) physics? In fact, there is no reason to expect global symmetries to be exact, but even a tiny explicit breaking of $\mathrm{U}(1)_{\mathrm{PQ}}$ in the UV would spoil the PQ solution to the strong $\mathrm{CP}$ problem. Although the PQ quality problem is eventually a matter of UV physics which cannot be definitively assessed without a calculable theory of quantum gravity, the 
requirement of having the $\mathrm{U}(1)_{\mathrm{PQ}}$ to arise accidentally is a bare minimum that a sensible PQ theory in should structurally achieve.

It is the purpose of this work to revisit this question in the context of $\mathrm{SO}(10)$ GUTs. Since the $\mathrm{SO}(10)$ symmetry, by itself, is not sufficient to provide an automatic $\mathrm{U}(1)_{\mathrm{PQ}}$, some ingredient must be clearly added. An interesting possibility, put forth by Chang and Senjanovič [15], is to employ the $\mathrm{SU}(3)_{f}$ flavour symmetry of $\mathrm{SO}(10)$ (for related approaches without GUTs, see $[16,17])$. Remarkably, if the $\mathrm{SO}(10)$ representations are properly selected, the simultaneous presence of the horizontal $\left(\mathrm{SU}(3)_{f}\right)$ and vertical $(\mathrm{SO}(10))$ symmetry leads to an automatic PQ symmetry. While ref. [15] focussed on the case of global $\mathrm{SU}(3)_{f}$, motivated by the possibility of testing the $\mathrm{SU}(3)_{f}$ breaking dynamics at low-energy via the associated Goldstone bosons (familons), from a modern perspective it would be more satisfactory to get a $\mathrm{U}(1)_{\mathrm{PQ}}$ symmetry to arise from local $\mathrm{SU}(3)_{f}$. The main obstacle for such a program is the cancellation of the $\mathrm{SU}(3)_{f}^{3}$ gauge anomaly, which requires to extend the fermion content of $\mathrm{SO}(10)$. In this work, we show how this can be consistently done, and we extend the analysis of [15] in several respects: $i$ ) we point out that the same approach can be used beyond the renormalizable level, thus providing also a way to tackle the PQ quality problem; $i i$ ) we identify the physical axion field and compute its low-energy couplings to SM matter fields. In this step, we realized that the charge assignment of [15] must be slightly modified, in order to avoid an alignment between two $\mathrm{SO}(10)$ Higgs representations which would otherwise lead to a Weinberg-Wilczek axion; iii) we address $\mathrm{SU}(3)_{f}$ breaking dynamics.

A relevant phenomenological feature of the accidental $\mathrm{SO}(10)$ axion is that its decay constant is bounded from above by the seesaw scale, which can be translated into a lower bound on the axion mass, $m_{a} \gtrsim 7 \times 10^{-8} \mathrm{eV}$. In fact, as we are going to show, in order to get an automatic PQ symmetry the $\mathrm{SO}(10)$ representations responsible for $\mathrm{U}(1)_{\mathrm{PQ}}$ breaking need to have a non-trivial $\mathrm{SO}(10)$ center. This immediately rules out the GUT-scale axion $\left(m_{a} \lesssim 10^{-9} \mathrm{eV}\right)$, in which the PQ breaking is connected to the first stage of $\mathrm{SO}(10)$ breaking, and selects instead $\mathrm{SO}(10)$ representations with $B-L$ breaking vacuum expectation values (VEVs) at intermediate mass scales well below GUT. Other phenomenological constraints on the axion mass depend on whether the PQ is broken before or after inflation. In the former case, an upper bound of about $m_{a} \lesssim 10^{-3} \mathrm{eV}$ originates from iso-curvature fluctuations generated by the massless axion field during inflation. On the other hand, a high-quality PQ symmetries requires a relatively heavy axion, $m_{a} \gtrsim 0.02 \mathrm{eV}$, that is viable only if the PQ symmetry is broken after inflation and never restored afterwards. This scenario is threatened by a genuine domain wall problem, which however can be overcome by a small explicit breaking of the $\mathrm{U}(1)_{\mathrm{PQ}}$ (in terms of Planck-suppressed operators), compatibly with the PQ solution of the strong CP problem. We also discuss the relevance of astrophysical bounds in such case and the possibility that DM is wholly comprised by axions in the high-quality axion mass window.

The paper is structured as follows. In section 2 we show how to obtain an accidental $\mathrm{U}(1)_{\mathrm{PQ}}$ symmetry upon gauging the flavour group of $\mathrm{SO}(10)$ and specify a minimal realistic model. In section 3 we extend the analysis beyond the renormalizable level, in order to identify at which operator level the $\mathrm{U}(1)_{\mathrm{PQ}}$ gets broken. Here, we also comment on the 
physical relevance of the PQ quality problem and on the possibility to address it within the present framework. Section 4 is devoted to the identification of the physical axion field, which requires some care due to the fact that the axion gets kinetically mixed with neutral massive vectors arising from $\mathrm{SO}(10)$ breaking. Bringing the axion in a canonical form is actually a necessary step for computing its low-energy couplings to SM matter fields (as detailed in appendix A). Next, in section 5 we address the cancellation of the $\mathrm{SU}(3)_{f}^{3}$ gauge anomaly as well as $\mathrm{SU}(3)_{f}$ breaking dynamics. In section 6 we discuss the phenomenological profile of the accidental $\mathrm{SO}(10)$ axion and conclude in section 7 , with a general perspective on the question of an automatic $\mathrm{U}(1)_{\mathrm{PQ}}$ in GUTs.

\section{Gauging the way to the $\mathrm{SO}(10)$ axion}

A family of SM fermions plus a right-handed neutrino reside into a spinorial $\mathrm{SO}(10)$ representation, $\psi_{16}$, which gets triplicated in order to account for three SM chiral families. Schematically, a typical SO(10) Yukawa Lagrangian reads

$$
\mathscr{L}_{Y}=y_{10} \psi_{16} \psi_{16} \phi_{10}+\tilde{y}_{10} \psi_{16} \psi_{16} \phi_{10}^{\star}+y_{\overline{126}} \psi_{16} \psi_{16} \phi_{\overline{126}}+\text { h.c. },
$$

where we restricted for simplicity to a $10+\overline{126}$ reducible Higgs representation, and we have taken a complex $\phi_{10}$ (as required by realistic fermion masses and mixings [18-21]), while the anti self-dual $\phi_{\overline{126}}$ is by construction complex. ${ }^{1}$

In the $y_{10}, \tilde{y}_{10}, y_{\overline{126}} \rightarrow 0$ limit the global symmetry group of the $\mathrm{SO}(10)$ fermion sector comprising three copies of $\psi_{16}$ is

$$
\mathrm{U}(3)=\mathrm{U}(1)_{\mathrm{PQ}} \times \mathrm{SU}(3)_{f},
$$

where the abelian factor is a PQ symmetry, since it is anomalous under QCD. In fact, the chiral embedding of SM matter into the spinorial of $\mathrm{SO}(10)$ implies a non-zero $\left.\mathrm{U}_{(1}\right)_{\mathrm{PQ}^{-}}$ $\mathrm{SU}(3)_{c}^{2}$ anomaly (cf. section 4).

In the following, we wish to argue that the gauging of $\mathrm{SU}(3)_{f}$ leads to an accidental $\mathrm{U}(1)_{\mathrm{PQ}}$ in the full Lagrangian, if the $\mathrm{SO}(10)$ Higgs representations are properly chosen along the lines of [15]. Since the $\mathrm{SO}(10)$ spinors transform in the fundamental of $\mathrm{SU}(3)_{f}$, $\psi_{16} \sim \mathbf{3}$, in order to make the Yukawa Lagrangian $\mathrm{SU}(3)_{f}$ invariant we need to assign both $\phi_{10} \sim \overline{\mathbf{6}}$ and $\phi_{\overline{126}} \sim \overline{\mathbf{6}}$, since $\mathrm{SO}(10)$ contractions are symmetric. ${ }^{2}$ Then the $\tilde{y}_{10}$ term in eq. (2.1) is forbidden by $\mathrm{SU}(3)_{f}$ gauge invariance and a $\mathrm{U}(1)_{\mathrm{PQ}}$ automatically arises in the Yukawa sector:

$$
\psi_{16} \rightarrow e^{i \alpha} \psi_{16}, \quad \phi_{10, \overline{126}} \rightarrow e^{-2 i \alpha} \phi_{10, \overline{126}},
$$

where $\alpha$ indicates the parameter of the $\mathrm{U}(1)_{\mathrm{PQ}}$ transformation and $\mathrm{PQ}$ charges are explicitly denoted as $\mathrm{PQ}\left(\psi_{16}\right)=1, \mathrm{PQ}\left(\phi_{10, \overline{126}}\right)=-2$, etc.

Other Higgs representations need to be introduced for the spontaneous symmetry breaking of $\mathrm{SO}(10) \times \mathrm{U}(1)_{\mathrm{PQ}}\left(\mathrm{SU}(3)_{f}\right.$ breaking will be discussed separately in section 5.2). To this end we consider a (real) adjoint $\phi_{45}$ and a $\phi_{16}$. Their role is the following:

\footnotetext{
${ }^{1}$ The reader not familiar with $\mathrm{SO}(10)$ properties can find a basic introduction e.g. in section 2 of [22].

${ }^{2}$ On the contrary, a $\phi_{120}$ would have to be assigned to a $\mathbf{3}$, being the $\psi_{16} \psi_{16} \phi_{120} \mathrm{SO}(10)$ contractions antisymmetric.
} 
- $\phi_{45}$ : this is the smallest representation which can break $\mathrm{SO}(10)$ down to a rank-5 sub-group featuring an unbroken $\mathrm{U}(1)_{R} \times \mathrm{U}(1)_{B-L}$ Cartan subalgebra, although it requires to go beyond the tree approximation for the minimization of the scalar potential [23-26]. As far as concerns the transformation properties of $\phi_{45}$ under SU(3) ${ }_{f}$, since it does not couple to $\psi_{16}$ at the renormalizable level, it can be taken to be a singlet. In fact, even if we were to assign $\phi_{45}$ to a non-trivial SU $(3)_{f}$ representation (say a fundamental), a PQ breaking operator of the type $\phi_{45}^{3} \equiv \epsilon_{(3)}^{a b c}\left(\phi_{45}\right)_{a}^{i j}\left(\phi_{45}\right)_{b}^{j k}\left(\phi_{45}\right)_{c}^{k i}$ would be always allowed by $\mathrm{SO}(10) \times \mathrm{SU}(3)_{f}$ invariance. Similar considerations apply to other $\mathrm{SO}(10)$ representations with a trivial $\mathrm{SO}(10)$ center, like 54 and 210, which could be similarly employed in place of $\phi_{45}$.

- $\phi_{16}$ : the need for this extra representation [27] is due to the fact that $\phi_{\overline{126}}$ breaks $\mathrm{U}(1)_{R} \times \mathrm{U}(1)_{B-L} \times \mathrm{U}(1)_{\mathrm{PQ}} \rightarrow \mathrm{U}(1)_{\mathrm{PQ}}^{\prime} \times \mathrm{U}(1)_{Y}$, where $\mathrm{U}(1)_{\mathrm{PQ}}^{\prime}$ is a remnant PQ symmetry (a linear combination of the original PQ and the broken gauge generators) that would be eventually broken at the electroweak scale, leading to a phenomenologically untenable Weinberg-Wilczek axion. Hence, the $\phi_{16}$ (together with $\phi_{\overline{126}}$ ) is needed to ensure a proper rank reduction down to the SM group. In order for the $\phi_{16}$ to participate to $\mathrm{U}(1)_{\mathrm{PQ}}$ breaking, it needs to couple in a non-trivial way to $\phi_{10}$ and/or $\phi_{\overline{126}}$ so that it can get charged under the PQ. The simplest, viable option is that $\phi_{16} \sim \overline{\mathbf{3}}$, thus allowing for the gauge invariant operator $\phi_{16}^{2} \phi_{10}^{\star}\left(\phi_{45}\right)$ (the parenthesis meaning both the invariant with and without $\left.\phi_{45}\right)$, so that $\mathrm{PQ}\left(\phi_{16}\right)=-1 .^{3}$

Summarizing, the Higgs sector includes $\phi_{10} \sim \overline{\mathbf{6}}, \phi_{\overline{126}} \sim \overline{\mathbf{6}}, \phi_{45} \sim \mathbf{1}$ and $\phi_{16} \sim \overline{\mathbf{3}}$. Then the scalar potential, $\mathcal{V}=\mathcal{V}_{2}+\mathcal{V}_{3}+\mathcal{V}_{4}$ (with the subscript denoting the dimensionality of the operators), features the following $\mathrm{SO}(10) \times \mathrm{SU}(3)_{f}$ invariant terms

$$
\begin{aligned}
& \mathcal{V}_{2}=\left|\phi_{10}\right|^{2}+\left|\phi_{126}\right|^{2}+\phi_{45}^{2}+\left|\phi_{16}\right|^{2} \\
& \mathcal{V}_{3}=\phi_{16}^{2} \phi_{10}^{\star}+\text { h.c. } \\
& \mathcal{V}_{4}=\mathcal{V}_{2}^{2} \text {-terms }+\phi_{10}^{2} \phi_{\frac{2}{126}}+\phi_{10} \phi_{\overline{126}} \phi_{\frac{2}{126}}+\phi_{16}^{2} \phi_{10}^{\star} \phi_{45}+\text { h.c. }
\end{aligned}
$$

where $\mathcal{V}_{2}^{2}$-terms stands for quartics obtained by "squaring" $\mathcal{V}_{2}$ (including all possible linearly independent invariants made by the same amount of fields). Note that the operator $\phi_{16}^{2} \phi_{126}^{\star}\left(\phi_{45}\right)$ is not allowed by $\mathrm{SO}(10)$ invariance, but due to the interplay with the $\phi_{10}$ there is a sufficient amount of scalar potential terms so that a single abelian global symmetry survives accidentally. This can be identified with the $\mathrm{U}(1)_{\mathrm{PQ}}$, with transformation properties

$$
\phi_{16} \rightarrow e^{-i \alpha} \phi_{16}, \quad \phi_{45} \rightarrow \phi_{45},
$$

while those of $\phi_{10}$ and $\phi_{\overline{126}}$ are given in eq. (2.3). The $\mathrm{SU}(3)_{f}$ symmetry forbids the following terms which are allowed by $\mathrm{SO}(10)$ invariance:

$$
\phi_{10}^{2}, \phi_{10}^{4}, \phi_{16}^{4}, \phi_{126}^{4}, \phi_{10}^{2} \phi_{45}^{2}, \phi_{10}^{2} \phi_{126}^{2}, \phi_{10} \phi_{\frac{3}{126}}^{3}, \phi_{45}^{2} \phi_{126}^{2}, \phi_{16}^{2} \phi_{10}\left(\phi_{45}\right), \phi_{16}^{2} \phi_{\overline{126}}\left(\phi_{45}\right) \text {. }
$$

\footnotetext{
${ }^{3}$ Refs. [15, 27] considered instead the case in which the following operators $\phi_{16}^{2} \phi_{10}\left(\phi_{45}\right)$ and $\phi_{16}^{2} \phi_{\overline{126}}\left(\phi_{45}\right)$ are present, and hence $\mathrm{PQ}\left(\phi_{16}\right)=1$. As we are going to show in section 4 (cf. footnote (6)), the latter choice implies an alignment between $\left\langle\phi_{16}\right\rangle$ and $\left\langle\phi_{\overline{126}}\right\rangle$, ending up in a Weinberg-Wilczek axion.
} 


\begin{tabular}{|c|c|cc|cc|c|}
\hline Field & Lorentz & $\mathrm{SO}(10)$ & $\mathbb{Z}_{4}$ & $\mathrm{SU}(3)_{f}$ & $\mathbb{Z}_{3}$ & $\mathrm{U}(1)_{\mathrm{PQ}}$ \\
\hline$\psi_{16}$ & $(1 / 2,0)$ & 16 & $i$ & $\mathbf{3}$ & $e^{i 2 \pi / 3}$ & 1 \\
$\psi_{1}^{1, \ldots, 16}$ & $(1 / 2,0)$ & 1 & 1 & $\overline{\mathbf{3}}$ & $e^{i 4 \pi / 3}$ & 0 \\
\hline$\phi_{10}$ & $(0,0)$ & 10 & -1 & $\overline{\mathbf{6}}$ & $e^{i 2 \pi / 3}$ & -2 \\
$\phi_{16}$ & $(0,0)$ & 16 & $i$ & $\overline{\mathbf{3}}$ & $e^{i 4 \pi / 3}$ & -1 \\
$\phi_{\overline{126}}$ & $(0,0)$ & $\overline{126}$ & -1 & $\overline{\mathbf{6}}$ & $e^{i 2 \pi / 3}$ & -2 \\
$\phi_{45}$ & $(0,0)$ & 45 & 1 & $\mathbf{1}$ & 1 & 0 \\
\hline
\end{tabular}

Table 1. Field content of the model and relative transformation properties under $\mathrm{SO}(10) \times \mathrm{SU}(3)_{f}$, its $\mathbb{Z}_{4} \times \mathbb{Z}_{3}$ center and the accidental $\mathrm{U}(1)_{\mathrm{PQ}}$. In light gray, exotic fermions which ensure $\mathrm{SU}(3)_{f}^{3}$ anomaly cancellation (cf. section 5.1).

The origin of the accidental $\mathrm{U}(1)_{\mathrm{PQ}}$ can be neatly understood in terms of the action of the centers of $\mathrm{SO}(10)$ and $\mathrm{SU}(3)_{f}$, which are respectively $\mathbb{Z}_{4}$ and $\mathbb{Z}_{3} \cdot{ }^{4}$ The transformation properties of the model fields under the gauge and the $\mathrm{U}(1)_{\mathrm{PQ}}$ accidental symmetries are summarized in table 1.

Note that for consistency we have also introduced 16 ( $\mathrm{SO}(10)$-singlet) exotic fermions in the $\overline{\mathbf{3}}$ of $\mathrm{SU}(3)_{f}$, to ensure $\mathrm{SU}(3)_{f}^{3}$ anomaly cancellation. Their spectrum will be discussed in section 5 , together with $\mathrm{SU}(3)_{f}$ breaking.

\section{Peccei-Quinn quality}

After having obtained the $\mathrm{U}(1)_{\mathrm{PQ}}$ to arise accidentally in the renormalizable Lagrangian, one should worry about possible sources of PQ breaking in the UV, which are often parametrized via effective operators suppressed by a cut-off scale $\Lambda_{\mathrm{UV}}$. A simple estimate shows that $\mathrm{U}(1)_{\mathrm{PQ}}$ should be preserved by operators up to dimension $d \gtrsim 9$, assuming for instance $\Lambda_{\mathrm{UV}} \sim M_{\mathrm{Pl}}$ and an axion decay constant $f_{a} \sim 10^{9} \mathrm{GeV}$. This is obtained by requiring that the energy density from UV sources of $\mathrm{PQ}$ breaking is about $10^{-10}$ times smaller than the energy density of the QCD axion potential

$$
\left(\frac{f_{a}}{\Lambda_{\mathrm{UV}}}\right)^{d-4} f_{a}^{4} \lesssim 10^{-10} \Lambda_{\mathrm{QCD}}^{4}
$$

so that the induced axion VEV displacement from zero is $\langle a\rangle / f_{a} \lesssim 10^{-10}$, within the bound from the neutron electric dipole moment (nEDM).

Interestingly, the gauging of $\mathrm{SU}(3)_{f}$ provides some protection also beyond the renormalizable level. Given the field content in table 1, we proceed to identify at which operator level the $\mathrm{U}(1)_{\mathrm{PQ}}$ gets broken in the scalar potential. The lowest-dimensional PQ-breaking

\footnotetext{
${ }^{4}$ The center $Z(G)$ of a group $G$ is the set of elements that commute with every element of $G$. $Z(\mathrm{SU}(3))=$ $\mathbb{Z}_{3}$, which is generated by $e^{2 \pi i / 3} \mathbb{1}_{3} ; Z(\mathrm{SO}(10))=\mathbb{Z}_{4}$, which is generated by $i \Gamma_{0}$, with $\Gamma_{0}$ denoting the "chirality" operator of the $\mathrm{SO}(10)$ Clifford algebra (see e.g. [37]).
} 
operators are found to be:

$$
\begin{array}{ll}
\phi_{10}^{6} & (d=6), \\
\phi \frac{6}{126} & (d=6), \\
\phi_{16}^{6} \phi_{10}^{3} & (d=9), \\
\phi_{16}^{6} \phi_{\overline{126}}^{3} & (d=9), \\
\phi_{16}^{12} & (d=12) .
\end{array}
$$

This classification can be easily understood in terms of the action of the $\mathbb{Z}_{4} \times \mathbb{Z}_{3}$ center as displayed in table 1: invariance under $\mathbb{Z}_{3}$ requires a number of fields that is a multiple of 3 and after that one has to compensate powers in order to get a $\mathbb{Z}_{4}$ singlet. Note that the $d=9$ operators $\phi_{16}^{6} \phi_{10}^{\star 3}$ and $\phi_{16}^{6} \phi_{\frac{126}{26}}$ are also allowed by gauge invariance, but they preserve $\mathrm{U}(1)_{\mathrm{PQ}}$.

Some comments are in order, regarding the impact of those operators on the PQ quality problem:

- $\phi_{10}$ can only develop electroweak scale VEVs, hence the Planck-suppressed operators $\phi_{10}^{6}$ and $\phi_{16}^{6} \phi_{10}^{3}$ do not pose a problem for the PQ quality issue. Then, we only need to worry about operators developing "large" VEVs compared to the electroweak scale, i.e. $\phi_{16}$ and $\phi_{\overline{126}}$.

- Remarkably, the operators $\phi \frac{6}{126}$ and $\phi_{16}^{12}$ do not yield a large contribution to the axion potential, since (once projected on the SM vacuum) extra electroweak VEV insertions are needed. To see this, recall the decompositions under $\mathrm{SO}(10) \rightarrow \mathrm{SU}(5) \times \mathrm{U}(1)_{Z}[28]$ : $16 \rightarrow 1(-5)+\ldots$ and $\overline{126} \rightarrow 1(+10)+\ldots$, along the $\mathrm{SU}(5)$ singlet components. Clearly, $\phi \frac{6}{126}$ and $\phi_{16}^{12}$ have zero projection on $V_{126}^{6}$ and $V_{16}^{12}$ (the latter denoting the $\mathrm{SU}(5)$-singlet VEVs), since it is not possible to compensate the $\mathrm{U}(1)_{Z}$ charge.

- The leading contribution to the axion potential is then given by the operator $\phi_{16}^{6} \phi_{126}^{3}$, with a non-zero projection on the high-scale VEVs $V_{16}^{6} V_{\frac{1}{126}}^{3}$. In order to assess the impact of that PQ-breaking operator on the axion potential, one has still to identify the physical axion field. This step will be done in detail section 4, but we anticipate here the results for the assessment of the PQ quality problem. Using eq. (4.16) we find the following contribution to the axion potential:

$$
\mathcal{V}_{\mathrm{PQ}-\text { break }}=k \frac{\phi_{16}^{6} \phi \frac{3}{126}}{M_{\mathrm{Pl}}^{5}}+\text { h.c. } \supset \frac{|k|}{2^{7 / 2}} \frac{V_{16}^{6} V_{\frac{3}{126}}^{3}}{M_{\mathrm{Pl}}^{5}} \cos \left(\frac{a}{V / 12}+\delta\right),
$$

with $\delta=\operatorname{Arg} k$ denoting a generic $\mathcal{O}(1)$ phase and $V$ a function of $V_{16, \overline{126}}$ defined in eq. (4.18) (for $c_{1}=1$ ). Considering the total axion potential $\mathcal{V}=\mathcal{V}_{\mathrm{PQ}-\text { break }}+\mathcal{V}_{\mathrm{QCD}}$, with $\mathcal{V}_{\mathrm{QCD}}=-\Lambda_{\mathrm{QCD}}^{4} \cos \left(a / f_{a}\right)$ and $f_{a}$ given in terms of $V_{16, \overline{126}}$ via eq. (4.20), one finds the induced QCD theta term (valid for $\langle a\rangle / f_{a} \ll 1$ ):

$$
\theta_{\mathrm{eff}} \equiv \frac{\langle a\rangle}{f_{a}} \approx \frac{|k| V_{16}^{6} V_{\frac{3}{126}}^{3} \sin \delta}{8 \sqrt{2} M_{\mathrm{Pl}}^{5} \Lambda_{\mathrm{QCD}}^{4}} .
$$


Requiring the neutron electric dipole moment bound, $\left|\theta_{\text {eff }}\right| \lesssim 2.0 \times 10^{-10}$, implies $\left(V_{16}^{3} V_{126}^{6}\right)^{1 / 9} \lesssim 2.0 \times 10^{9} \mathrm{GeV}$ (for e.g. $|k|=1$ and $\sin \delta=1$ ). A more useful estimate in terms of the axion decay constant will be given in section 6 .

- Last, but not least, assuming no new physics above the GUT scale apart for gravity, it is unclear whether quantum gravity would generate power-like PQ-breaking operators, such as those in eq. (3.7). Nonetheless, it is believed that gravity does violate global symmetries, based on semi-classical arguments related to black holes and Hawking radiation. In scenarios in which Einstein gravity is minimally coupled to the axion field, non-conservation of the PQ global charge arises from non-perturbative effects described by Euclidean wormholes. Those are calculable in the semi-classical limit [29-33] and give a correction to the axion potential of the order of $M_{\mathrm{Pl}}^{4} e^{-S_{\mathrm{wh}}}$, where $S_{\mathrm{wh}} \sim M_{\mathrm{Pl}} / f_{a}$ is the wormhole action. Being exponentially suppressed, such contribution poses a problem for the PQ solution only for $f_{a} \gtrsim 10^{17} \mathrm{GeV}$, which is however much above the intermediate-scale values of $f_{a}$ that will be considered here.

All in all, we conclude that not only the gauging of $\mathrm{SU}(3)_{f}$ leads to an accidental $\mathrm{U}(1)_{\mathrm{PQ}}$, but it also structurally protects it against PQ breaking effective operators. To solve the PQ quality issue in its standard formulation in terms of Planck-suppressed, power-like effective operators one needs PQ breaking VEVs smaller than about $10^{9} \mathrm{GeV}$.

\section{Axion's guts (physical axion field)}

A crucial quantity that qualifies the $\mathrm{U}(1)$ accidental symmetry as a PQ symmetry is the QCD anomaly of the classically conserved PQ current, $J_{\mu}^{\mathrm{PQ}}$. At the $\mathrm{SO}(10)$ level, that is (employing the compact notation $G \tilde{G} \equiv G_{\mu \nu}^{a} \tilde{G}^{a \mu \nu}$, etc.)

$$
\begin{aligned}
\partial^{\mu} J_{\mu}^{\mathrm{PQ}} & =\frac{\alpha_{\mathrm{SO}(10)} N_{\mathrm{SO}(10)}}{4 \pi} F_{\mathrm{SO}(10)} \tilde{F}_{\mathrm{SO}(10)} \\
& \supset \frac{\alpha_{s} N_{\mathrm{SO}(10)}}{4 \pi} \tilde{G}+\frac{\alpha_{W} N_{\mathrm{SO}(10)}}{4 \pi} W \tilde{W}+\frac{5}{3} \frac{\alpha_{Y} N_{\mathrm{SO}(10)}}{4 \pi} B \tilde{B} \\
& \supset \frac{\alpha_{s} N_{\mathrm{SO}(10)}}{4 \pi} G \tilde{G}+\frac{8}{3} \frac{\alpha N_{\mathrm{SO}(10)}}{4 \pi} F \tilde{F} \equiv \frac{\alpha_{s} N}{4 \pi} G \tilde{G}+\frac{\alpha E}{4 \pi} F \tilde{F},
\end{aligned}
$$

where the matching with the SM gauge bosons is understood at the GUT scale. The matching with QED is obtained by projecting on the photon component ( $W^{3} \rightarrow \sin \theta_{W} A$ and $\left.B \rightarrow \cos \theta_{W} A\right)$ and using $\alpha=\alpha_{W} \sin ^{2} \theta_{W}=\alpha_{Y} \cos ^{2} \theta_{W}$. In the last step we made contact with the standard notation for the QCD $(N)$ and QED $(E)$ anomaly coefficients, which read respectively $N=N_{\mathrm{SO}(10)}$ and $E=8 / 3 N_{\mathrm{SO}(10)}$, in terms of the $\mathrm{SO}(10)$ anomaly factor

$$
N_{\mathrm{SO}(10)}=n_{g} T\left(\psi_{16}\right) \mathrm{PQ}\left(\psi_{16}\right)=6,
$$

with $n_{g}=3$ (number of generations), $T\left(\psi_{16}\right)=2$ (Dynkin index of the spinorial of SO(10)) and $\mathrm{PQ}\left(\psi_{16}\right)=1$. Upon the anomalous PQ rotation $\psi_{16} \rightarrow e^{i \alpha} \psi_{16}$, the QCD theta term in $\mathscr{L}_{\mathrm{QCD}} \supset \frac{\alpha_{s} \theta}{8 \pi} G \tilde{G}$ is shifted as $\theta \rightarrow \theta+2 N \alpha$. Since different values of $\theta$ correspond to 
different physics modulo $2 \pi$, the $\mathrm{U}(1)_{\mathrm{PQ}}$ symmetry is explicitly broken down to a $\mathbb{Z}_{2 N}$, corresponding to $\alpha=2 \pi n /(2 N)$ with $n=0,1, \ldots, 2 N-1$.

The anomaly does not depend on the mass of the fermions running in the triangle loop, and hence it must be preserved through the various stages of symmetry breaking. This feature will be useful for identifying the physical axion field and its low-energy couplings. In the following, we provide the anatomy of the axion field in $\mathrm{SO}(10)$ at three different levels of increasing complexity: $i$ ) a single, dominant SM-singlet and PQ-breaking VEV, ii) two SM-singlet and PQ-breaking VEVs of comparable size and iii) the full-fledged case including also electroweak VEVs, a necessary step to compute low-energy axion couplings to SM matter fields. The last, self-contained derivation is deferred to appendix A.

\subsection{A dominant SM-singlet and PQ-breaking VEV}

Let us first consider a phenomenologically relevant limit where physics should be clearer, and focus on the breaking pattern

$$
\begin{aligned}
\mathrm{SO}(10) \times \mathrm{U}(1)_{\mathrm{PQ}} & \stackrel{\left\langle\phi_{45}\right\rangle_{B-L}}{\longrightarrow} \mathrm{SU}(3)_{c} \times \mathrm{SU}(2)_{L} \times \mathrm{SU}(2)_{R} \times \mathrm{U}(1)_{B-L} \times \mathrm{U}(1)_{\mathrm{PQ}} \\
& \stackrel{V_{126}}{\longrightarrow} \mathrm{SU}(3)_{c} \times \mathrm{SU}(2)_{L} \times \mathrm{U}(1)_{Y} \times \mathrm{U}(1)_{\mathrm{PQ}}^{\prime} \\
& \stackrel{V_{16}}{\longrightarrow} \mathrm{SU}(3)_{c} \times \mathrm{SU}(2)_{L} \times \mathrm{U}(1)_{Y},
\end{aligned}
$$

where we assumed the hierarchy of VEVs: $\left\langle\phi_{45}\right\rangle_{B-L} \gg V_{\overline{126}} \gg V_{16}$, as motivated by gauge coupling unification [34-36]. Here, $\left\langle\phi_{45}\right\rangle_{B-L}$ denotes a specific orientation of the adjoint VEV, which can be achieved via a renormalizable scalar potential after including one-loop corrections [23-26]; while the other two intermediate-scale VEVs are defined in terms of the following polar field decompositions (along the SM-singlet components)

$$
\phi_{16}=\frac{1}{\sqrt{2}}\left(V_{16}+\ldots\right) e^{i \frac{a_{16}}{V_{16}}}, \quad \phi_{\overline{126}}=\frac{1}{\sqrt{2}}\left(V_{\overline{126}}+\ldots\right) e^{i \frac{a_{\overline{126}}}{V_{126}}},
$$

with the projections of the angular modes $a_{16}$ and $a_{\overline{126}}$ on the axion field yet to be identified. After the second breaking stage, a remnant $\mathrm{U}(1)_{\mathrm{PQ}}^{\prime}$ global symmetry is left invariant by $\left\langle\phi_{\overline{126}}\right\rangle$ (which cannot reduce the rank by more than one unit). The latter symmetry can be expressed as a linear combination of the original $\mathrm{U}(1)_{\mathrm{PQ}}$ and the broken Cartan gauge generators $\mathrm{as}^{5}$

$$
\mathrm{PQ}^{\prime}=c_{1} \mathrm{PQ}+c_{2}(B-L) .
$$

Given $\{\mathrm{PQ}, B-L\}\left(\left\langle\phi_{\overline{126}}\right\rangle\right)=\{-2,-2\}$, the defining property, $\mathrm{PQ}^{\prime}\left(\left\langle\phi_{\overline{126}}\right\rangle\right)=0$, implies $c_{2}=-c_{1}$. The coefficient $c_{1}$ can be fixed by matching the anomaly in eq. (4.2) with the one computed in terms of $\mathrm{PQ}^{\prime}$ charges, that is

$$
N=n_{g} T(3)\left(2 \mathrm{PQ}^{\prime}(q)+2 \mathrm{PQ}^{\prime}\left(q^{c}\right)\right)=6 c_{1},
$$

where we used $n_{g}=3, T(3)=1 / 2$ (the Dynkin index of the fundamental of $\left.\mathrm{SU}(3)_{c}\right)$ and the $\mathrm{PQ}^{\prime}$ charges $\mathrm{PQ}^{\prime}(q)=2 c_{1} / 3$ (for the $\mathrm{SU}(2)_{L}$ quark doublet, with $B-L=1 / 3$ ) and

\footnotetext{
${ }^{5}$ Since at this point we only consider VEVs with zero hypercharge, $Y=T_{R}^{3}+(B-L) / 2$, it is not necessary to include the $T_{R}^{3}$ generator.
} 
$\mathrm{PQ}^{\prime}\left(q^{c}\right)=4 c_{1} / 3$ (for the $\mathrm{SU}(2)_{R}$ quark doublet, with $B-L=-1 / 3$ ). The matching with the $\mathrm{SO}(10)$ anomaly in eq. (4.2) requires then $c_{1}=1$, and hence $\mathrm{PQ}^{\prime}=\mathrm{PQ}-(B-L)$. Given $\{\mathrm{PQ}, B-L\}\left(\left\langle\phi_{16}\right\rangle\right)=\{-1,1\}$, it is readily verified that the action of $\mathrm{PQ}^{\prime}$ on $\left\langle\phi_{16}\right\rangle$ is non-trivial $\left(\mathrm{PQ}^{\prime}\left(\left\langle\phi_{16}\right\rangle\right)=-2\right)$ and hence it allows us to consistently break $\mathrm{U}(1)_{\mathrm{PQ}}^{\prime}$ at the scale $V_{16} \cdot{ }^{6}$ Neglecting $\left(V_{16} / V_{\overline{126}}\right)^{2}$ corrections as well as subleading electroweak VEVs, the axion field is identified with the angular component of the $\phi_{16}$ field, $a=a_{16}$.

In the other relevant limit, $V_{16} \gg V_{\overline{126}}$, one obtains that another linear combination $\mathrm{U}(1)_{\mathrm{PQ}}^{\prime \prime}$, defined as $\mathrm{PQ}^{\prime \prime}=\mathrm{PQ}+(B-L)$, is left invariant by $\left\langle\phi_{16}\right\rangle$, which is further broken by $\left\langle\phi_{\overline{126}}\right\rangle\left(\mathrm{PQ}^{\prime \prime}\left(\left\langle\phi_{\overline{126}}\right\rangle\right)=-4\right)$, so that the axion field is $a=a_{\overline{126}}$.

\subsection{Two SM-singlet and PQ-breaking VEVs}

The more general case with $V_{16} \sim V_{\overline{126}}$ must interpolate between the two limits above and it can be obtained as follows. Consider the classically conserved currents

$$
\begin{aligned}
J_{\mu}^{\mathrm{PQ}} & =q_{16} V_{16} \partial_{\mu} a_{16}+q_{\overline{126}} V_{\overline{126}} \partial_{\mu} a_{\overline{126}}, \\
J_{\mu}^{B-L} & =(B-L)_{16} V_{16} \partial_{\mu} a_{16}+(B-L)_{\overline{126}} V_{\overline{126}} \partial_{\mu} a_{\overline{126}},
\end{aligned}
$$

with gauge charges $(B-L)_{16}=1$ and $(B-L)_{\overline{126}}=-2$, and the physical PQ charges $q_{16, \overline{126}}$. The latter are linear combination of the PQ charges in table 1 and broken gauge generators, in general

$$
q=c_{1} \mathrm{PQ}+c_{2}(B-L)+c_{3} Y,
$$

(with $c_{3}=0$ as long as we consider only the SM-singlet VEVs $V_{16, \overline{126}}$ ). The $q$ charges can be fixed by requiring that the $\mathrm{PQ}$ and $B-L$ currents are orthogonal:

$$
q_{16} V_{16}^{2}-2 q_{\overline{126}} V_{126}^{2}=0,
$$

which ensures no kinetic mixings between the axion field and the $B-L$ massive gauge boson, thus providing a canonical axion field. The latter is defined as

$$
a=\frac{1}{V}\left(q_{16} V_{16} a_{16}+q_{\overline{126}} V_{\overline{126}} a_{\overline{126}}\right),
$$

with

$$
V^{2}=\left(q_{16}\right)^{2} V_{16}^{2}+\left(q_{\overline{126}}\right)^{2} V_{126}^{2},
$$

so that $J_{\mu}^{\mathrm{PQ}}=V \partial_{\mu} a$ and, compatibly with the Goldstone theorem, $\left\langle 0\left|J_{\mu}^{\mathrm{PQ}}\right| a\right\rangle=i V p_{\mu}$. Inverting the orthogonal transformation in eq. (4.11), one readily obtains the projection of the angular modes on the axion field:

$$
a_{16} \rightarrow q_{16} V_{16} \frac{a}{V}, \quad a_{\overline{126}} \rightarrow q_{\overline{126}} V_{\overline{126}} \frac{a}{V},
$$

whose weight factors are extracted from eq. (4.10) and (4.12), and can be conveniently written as

$$
q_{16} V_{16}=V \cos \omega, \quad q_{\overline{126}} V_{\overline{126}}=V \sin \omega,
$$

\footnotetext{
${ }^{6}$ Choosing instead the PQ charge of $\phi_{16}$ equal to 1 , leads to $\mathrm{PQ}^{\prime}\left\langle\phi_{16}\right\rangle=0$. Namely $\left\langle\phi_{16}\right\rangle$ and $\left\langle\phi_{\overline{126}}\right\rangle$ are aligned, thus not providing a proper breaking pattern for $\mathrm{U}(1)_{\mathrm{PQ}}^{\prime}$, that gets eventually broken at the electroweak scale.
} 
in terms of the vacuum parameter

$$
\tan \omega=\frac{V_{16}}{2 V_{\overline{126}}}
$$

So we can finally read the axion composition of the complex fields in eq. (4.4), which is

$$
\phi_{16}=\frac{1}{\sqrt{2}}\left(V_{16}+\ldots\right) e^{i \cos \omega \frac{a}{V_{16}}}, \quad \phi_{\overline{126}}=\frac{1}{\sqrt{2}}\left(V_{\overline{126}}+\ldots\right) e^{i \sin \omega \frac{a}{V_{126}}},
$$

For instance, taking the limit $V_{\overline{126}} \gg V_{16}(\omega \rightarrow 0)$ one reproduces the previous result that the axion dominantly correspond to the angular mode of $\phi_{16}$.

The orthogonality condition in eq. (4.10), together with the matching of the UV and IR charges of $\phi_{16}$ and $\phi_{\overline{126}}$ in eq. (4.9), allows us to fix

$$
c_{2}=-c_{1} \cos 2 \omega, \quad q_{16}=-2 c_{1} \cos ^{2} \omega, \quad q_{\overline{126}}=-4 c_{1} \sin ^{2} \omega,
$$

and hence, using eq. (4.12) (and eq. (4.14) regarding the absolute sign)

$$
V=-\frac{4 c_{1} V_{\overline{126}} V_{16}}{\sqrt{V_{16}^{2}+4 V_{126}^{2}}} .
$$

Again, the value of $c_{1}$ can be determined by matching the anomaly between the UV and the broken theory, that is

$$
N=n_{g} T(3)\left(2 q(q)+2 q\left(q^{c}\right)\right)=6 c_{1},
$$

where $q(q)=2 c_{1} / 3\left(\cos ^{2} \omega+2 \sin ^{2} \omega\right)$ and $q\left(q^{c}\right)=2 c_{1} / 3\left(2 \cos ^{2} \omega+\sin ^{2} \omega\right)$. Hence, $c_{1}=1$ in order to match the UV anomaly. The correct physical limits $V=-2 V_{16}$ (for $V_{\overline{126}} \rightarrow \infty$ ) and $V=-4 V_{\overline{126}}$ (for $V_{16} \rightarrow \infty$ ) are then recovered. ${ }^{7}$ Finally, the axion decay constant is

$$
f_{a}=\frac{V}{2 N}=-\frac{V_{\overline{126}} V_{16}}{3 \sqrt{V_{16}^{2}+4 V_{\overline{126}}^{2}}} .
$$

In the following, to ease the notation when discussing phenomenological bounds, we will refer to $f_{a}$ meaning its absolute value.

The most general case including also electroweak VEVs that participate to $\mathrm{U}(1)_{\mathrm{PQ}}$ breaking is discussed in appendix A, together with the derivation of low-energy axion couplings.

\subsection{Axion domain wall problem?}

The discrete $\mathbb{Z}_{2 N}$ symmetry left invariant by the QCD anomaly (cf. discussion below eq. (4.2)), implies that the axion potential has $2 N=12$ degenerate minima. At the QCD phase transition, this leads to the formation of domain-wall-like structures at the boundaries between regions of different vacua, which quickly dominate the energy density of the

\footnotetext{
${ }^{7}$ To see that, take for instance the $V_{\overline{126}} \rightarrow \infty$ limit, corresponding to $a \rightarrow a_{16}$. Then, given that under a PQ transformation $a_{16, \overline{126}} \rightarrow a_{16, \overline{126}}+\alpha q_{16, \overline{126}} V_{16, \overline{126}}$ the axion field transforms as $a \rightarrow a+\alpha V$ (cf. eq. (4.11)), we can make the identification $V=q_{16} V_{16} \rightarrow-2 V_{16}$.
} 
Universe. However, in the presence of extra global/local symmetries it could happen that some of those minima are connected. In particular, if the $\mathbb{Z}_{2 N}$ discrete symmetry can be fully embedded in the center of a non-abelian symmetry group, then the axion domain walls will quickly disappear through the emission of Goldstone bosons, thus providing an elegant solution to the axion domain wall problem [37]. Solutions of this type have been often considered in the context of $\mathrm{SO}(10)$ [37-39], whose $\mathbb{Z}_{4}$ center provides a starting point for embedding the $\mathbb{Z}_{2 N}$ discrete symmetry. This is possible in models where $2 N=4$ or if extra global/local symmetries beyond $\mathrm{SO}(10)$ are invoked (which is the case considered here).

While such a solution was claimed to be at play also in the $\mathrm{SO}(10) \times \mathrm{SU}(3)_{f}^{\text {global }}$ model of ref. [15], sharing several similarities with the present work, it turns out that this conclusion is flawed by the fact that in the model of [15] the PQ symmetries of $\phi_{16}$ and $\phi_{\overline{126}}$ were aligned, eventually leading to a phenomenologically untenable Weinberg-Wilczek axion (cf. the discussion in footnote (3)). After assigning the $\phi_{16}$ to a proper $\mathrm{SU}(3)_{f}$ representation (cf. table 1), so that its PQ charge is not aligned to the one of $\phi_{\overline{126}}$, it turns out that the remnant $\mathbb{Z}_{2 N}$ symmetry cannot be embedded in the center of $\mathrm{SO}(10) \times \mathrm{SU}(3)_{f}$ and hence the axion domain wall problem persists. To show this, let us define the action of the centers as $r_{P}=\exp (i 2 \pi / P)$. Denoting as $r_{12}$ the action of a PQ transformation with discrete parameter $\alpha=2 \pi / 12, r_{4}$ and $r_{3}$ the actions of the $\mathrm{SO}(10)$ and $\mathrm{SU}(3)_{f}$ centers (as displayed in table 1 ), one would like to have that $r_{12}=r_{4}^{-1} r_{3}$ on all the model fields, so that the two sets of symmetries can be identified. It is easy to check that while such identification works for the fields $\psi_{16}\left(r_{12}=r_{4}^{-1} r_{3}=\exp (i \pi / 6)\right), \phi_{10}, \phi_{\overline{126}}\left(r_{12}=r_{4}^{-1} r_{3}=\exp (-i \pi / 3)\right)$ and $\phi_{45}\left(r_{12}=r_{4}^{-1} r_{3}=1\right)$, it fails for $\phi_{16}\left(r_{12}=\exp (-i \pi / 6) \neq r_{4}^{-1} r_{3}=\exp (i 5 \pi / 6)\right)$ and $\psi_{1}^{1, \ldots, 16}\left(r_{12}=1 \neq r_{4}^{-1} r_{3}=\exp (i 2 \pi / 3)\right)$.

We hence conclude that the accidental $\mathrm{SO}(10)$ axion has a genuine domain wall problem, with domain wall number $N_{\mathrm{DW}} \equiv 2 N=12$. A straightforward solution is given by the pre-inflationary PQ breaking scenario (in which domain walls are inflated away). Instead, in the post-inflationary PQ breaking scenario the Planck-suppressed sources of explicit PQ breaking discussed in section 3 could potentially lift the vacuum degeneracy and lead to a fast decay of the domain walls [40,41], compatibly with the PQ solution of the strong CP problem. This possibility will be explored in more detail in section 6 .

\section{$5 \mathrm{SU}(3)_{f}$ sector}

We next discuss two issues related to the $\mathrm{SU}(3)_{f}$ sector, namely the cancellation gauge anomalies and the spontaneous symmetry breaking of $\mathrm{SU}(3)_{f}$.

\section{1 $\mathrm{SU}(3)_{f}^{3}$ anomaly}

The only relevant gauge anomaly to be cancelled is the $\mathrm{SU}(3)_{f}^{3}$ one (while $\mathrm{SO}(10)$ is anomaly free). The simplest way to cancel the latter is to introduce 16 exotic fermions, $\psi_{1}^{\alpha}(\alpha=1, \ldots, 16)$ which are $\mathrm{SO}(10)$ singlets and transform in the $\overline{\mathbf{3}}$ of $\mathrm{SU}(3)_{f} \cdot{ }^{8}$ Due to

\footnotetext{
${ }^{8}$ Other options to cancel the $\mathrm{SU}(3)_{f}^{3}$ anomaly include the following copies of $\mathrm{SO}(10)$-singlet representations: $2 \times \overline{\mathbf{6}}+2 \times \overline{\mathbf{3}}, 3 \times \overline{\mathbf{6}}+5 \times \mathbf{3}$, etc.
} 
their quantum numbers they have no renormalizable interactions with the other fields of table 1, so that they also acquire no PQ charge. Their spectrum is eventually controlled by $\mathrm{SU}(3)_{f}$ breaking, as discussed in the following. Let us also note that there is a remnant $\mathrm{U}(1)_{\mathrm{PQ}} \times \mathrm{SU}(3)_{f}^{2}$ anomaly (since only $\psi_{16}$ are charged under the $\mathrm{PQ}$ ). So, in order for the axion to relax to zero the $\theta$ term of QCD (and not the one of $\left.\mathrm{SU}(3)_{f}\right), \mathrm{SU}(3)_{f}$ should be spontaneously broken (effectively suppressing the contribution to the axion potential due to the Higgsing of $\mathrm{SU}(3)_{f}$ instantons). This is anyway compatible with the requirement that the flavour symmetry must be completely broken in order to give mass to SM fermions.

\section{$\mathbf{5 . 2} \mathrm{SU}(3)_{f}$ breaking}

We assume that $\mathrm{SU}(3)_{f}$ breaks completely, in two steps

$$
\mathrm{SU}(3)_{f} \stackrel{M_{1}}{\longrightarrow} \mathrm{SU}(2)_{f} \stackrel{M_{2}}{\longrightarrow} \mathbf{1}
$$

where 1 denotes the trivial group $\left(\mathrm{SU}(2)_{f}\right.$ completely broken). As suggested by SM charged fermion masses, we also assume a hierarchy of scales $M_{1} \gg M_{2}$. This could be achieved via two (misaligned) scalars transforming as $\phi^{1,2} \sim \overline{\mathbf{3}}$ or $\mathbf{6}$ of SU(3) $)_{f}$. At the same time, Yukawa operators $\psi_{1}^{\alpha} \psi_{1}^{\alpha} \phi^{1,2}, \psi_{1}^{\alpha} \psi_{1}^{\alpha}\left(\phi^{1,2}\right)^{2} / M_{\mathrm{Pl}}$, etc. (in the diagonal basis), would give a mass of order $M_{1,2}, M_{1,2}^{2} / M_{\mathrm{Pl}}$, etc. to the exotic fermions after complete $\mathrm{SU}(3)_{f}$ breaking. However, if $\phi^{1,2}$ were to transform under an $\mathrm{SO}(10)$ representation with trivial center, i.e. $1,45,54$ or 210, the following operators would be also allowed: $\phi_{10}^{2}\left(\phi^{1,2}\right)^{\star}, \phi_{10}^{2}\left(\phi^{1,2}\right)^{\star 2}$ and $\left(\phi^{1,2}\right)^{3}$, whose simultaneous presence would break explicitly the $\mathrm{U}(1)_{\mathrm{PQ}}$ at the renormalizable level. This can be avoided if we assigned instead $\phi^{1,2}$ e.g. to a 16 or $\overline{126}$.

A remarkably economical possibility (that is also unavoidable in absence of extra sources of $\mathrm{SU}(3)_{f}$ breaking at the GUT scale) is actually that of using the representations $\phi_{16}$ and $\phi_{\overline{126}}$ in table 1 to break simultaneously $\mathrm{SO}(10) \times \mathrm{U}(1)_{\mathrm{PQ}}$ and $\mathrm{SU}(3)_{f}$. There are no obvious obstructions to this program, which ties together the "horizontal" and "vertical" breaking, since the representations involved have the group-theoretical power to properly reduce the symmetry, ${ }^{9}$ while their high-scale VEVs would allow us to decouple the associated flavour breaking dynamics at safely large scales.

On the other hand, within such approach the 16 exotic fermions $\psi_{1}^{\alpha}$ remain massless at the renormalizable level. The following effective operators, involving a pair of exotic fermions are allowed by $\mathrm{SO}(10) \times \mathrm{SU}(3)_{f}$ invariance (omitting the $\alpha=1, \ldots, 16$ index)

$$
\frac{1}{M_{\mathrm{Pl}}} \psi_{1} \psi_{1} \phi_{10}^{2}, \quad \frac{1}{M_{\mathrm{Pl}}^{2}} \psi_{1} \psi_{1} \phi_{16}^{2} \phi_{\overline{126}}, \quad \frac{1}{M_{\mathrm{Pl}}^{3}} \psi_{1} \psi_{1} \phi_{16}^{4}, \quad \frac{1}{M_{\mathrm{Pl}}^{3}} \psi_{1} \psi_{1}\left(\phi_{\overline{126}}^{\star}\right)^{4} .
$$

Only the second operator gives a sizeable contribution to the mass of the exotic fermions, that are lifted up to $\left(V_{16}^{2} V_{\overline{126}}\right) / M_{\mathrm{Pl}}^{2} \lesssim 10 \mathrm{TeV}$ (for $V_{16, \overline{126}} \lesssim 10^{14} \mathrm{GeV}$ ), while the other operators have only projections on electroweak VEVs, thus providing a tiny contribution

\footnotetext{
${ }^{9}$ For instance, it should be clear that the VEV orientation $\left\langle\phi_{16}\right\rangle_{a=3}^{\alpha=16}$ breaks $\mathrm{SO}(10) \times \mathrm{SU}(3)_{f} \rightarrow \mathrm{SU}(5) \times$ $\mathrm{SU}(2)_{f}$, etc.
} 
to the mass of the exotic fermions. ${ }^{10}$ For lower values of $V_{16, \overline{126}}$ the exotic fermions are lighter and they might eventually contribute to dark radiation if once in thermal contact with the $\mathrm{SM}$ via $\mathrm{SU}(3)_{f}$ interactions.

Of course, it would be interesting to study whether the above flavour dynamics could be predictive for reproducing SM fermion masses and mixings. Here, we stress that the present approach to flavour differs from more standard ones (such as e.g. the one of ref. [42]), in which the Yukawas transform under $\mathrm{SU}(3)_{f}$, being themselves flavon fields interacting with SM fields via effective operators. In our case instead it is crucial, in order to obtain an automatic $\mathrm{U}(1)_{\mathrm{PQ}}$, that the flavour dynamics acts at the renormalizable level and that a single Higgs representation breaks both $\mathrm{SO}(10)$ and $\mathrm{SU}(3)_{f}$, which is closer in spirit to the approach of ref. [43]. A quantitative analyses of fermion masses and mixings following this path is left for future studies.

\section{Axion phenomenology}

In this section we describe the phenomenological profile of the accidental $\mathrm{SO}(10)$ axion. Axion couplings to photons and SM matter fields have been computed in appendix A (respectively eq. (A.26) and eqs. (A.30)-(A.31)). For the present scenario, the axion coupling to photons represents the main experimental probe (as shown in figure 1). The mass range of the accidental $\mathrm{SO}(10)$ axion is constrained by various considerations, depending on whether the PQ symmetry is broken before or after inflation.

\subsection{Pre-inflationary PQ breaking}

If the PQ symmetry is broken before (and during) inflation and not restored afterwards, the dark matter (DM) relic density via the vacuum misalignment mechanism is given by $[45,46]$

$$
\Omega_{a} h^{2}=0.12\left(\frac{f_{a}}{9 \times 10^{11} \mathrm{GeV}}\right)^{1.165} \theta_{\mathrm{in}}^{2},
$$

which is valid for $\theta_{\text {in }} \lesssim 1$. In the non-linear regime, $\theta_{\text {in }} \rightarrow \pi$, DM axions are allowed to have masses as high as $m_{a} \lesssim 1 \mathrm{meV}$, but no higher because quantum fluctuations during inflation would imply too large iso-curvature fluctuations [47].

On the other hand, a lower bound on $m_{a}$ follows as well within the model, since $f_{a}$ is bounded from above by the seesaw scale. Rewriting eq. (4.20) as $\left|f_{a}\right|=|\sin 2 \omega| V_{B-L} / 12$, with $V_{B-L} \equiv \sqrt{V_{16}^{2}+4 V_{126}^{2}}$, we find the upper limit $f_{a} \leq V_{B-L} / 12$. Note that the mass of the $B-L$ gauge boson will be proportional to $V_{B-L}$ (because of the relative $B-L$ charges of the two VEVs). Hence, $V_{B-L}$ assumes the meaning of $B-L$ breaking scale, subject to

\footnotetext{
${ }^{10}$ One might worry that the operators in eq. (5.2) break the PQ symmetry and hence do contribute to the axion potential upon closing fermion loops. Note, however, that it is possible to make at least one of those operators formally $\mathrm{PQ}$ invariant, by a proper assignment of the PQ charge of the exotic fermions, respectively $\mathrm{PQ}\left(\psi_{1}\right)=\{-2,2,2,-4\}$, so that the simultaneous presence of at least two of them is required in order to genuinely break the PQ symmetry. The largest contribution comes by combining the second operator, involving a SM-singlet VEV, with another operator that necessarily involves an electroweak scale VEV. The latter suppression makes the contribution to the axion potential safely negligible.
} 


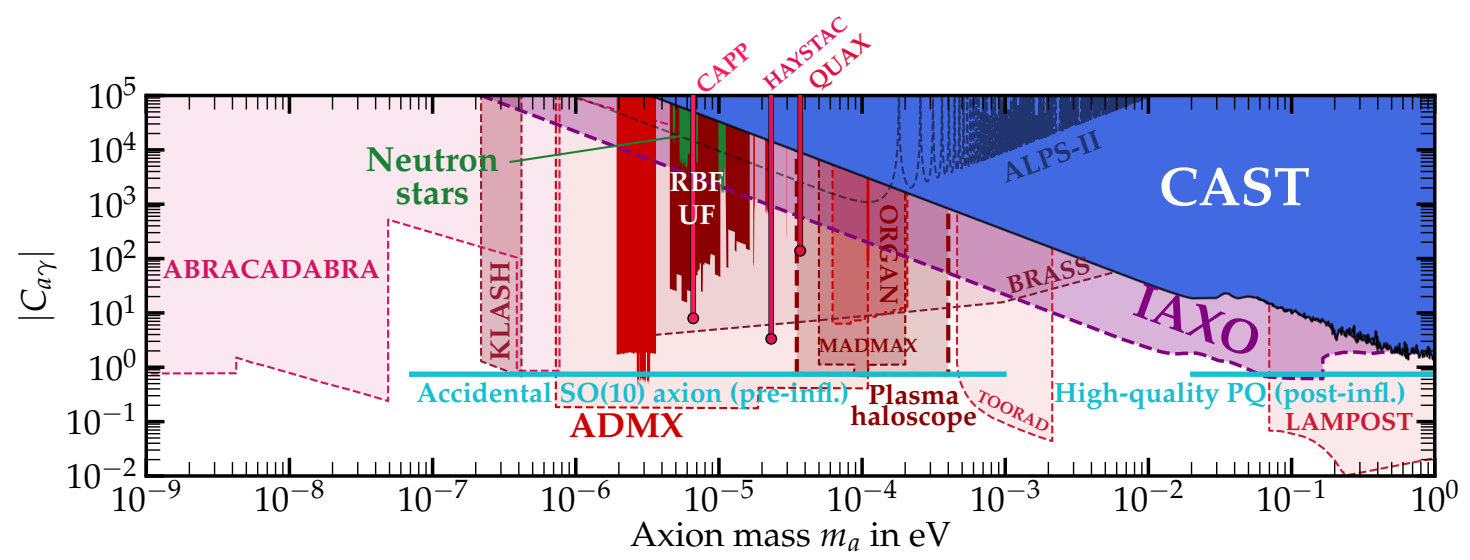

Figure 1. Axion-photon coupling (eq. (A.26) with $E / N=8 / 3$ ) and sensitivity of present (full lines) and future (dashed lines) axion experiments. The accidental $\mathrm{SO}(10)$ axion mass window (in the pre-inflationary PQ breaking scenario) corresponds to $m_{a} \in\left[7 \times 10^{-8}, 10^{-3}\right] \mathrm{eV}$. A high-quality PQ symmetry is obtained for $m_{a} \gtrsim 0.02 \mathrm{eV}$ (in the post-inflationary PQ breaking scenario). Axion limits from [44].

constraints both from gauge coupling unification and neutrino masses. In particular, righthanded neutrino masses are controlled at tree-level by $V_{\overline{126}}$, via $M_{\nu_{R}}=y_{\overline{126}} V_{\overline{126}} / \sqrt{2}$, and at two loops $[48,49]$ by $V_{16}$. Very conservatively, we take $V_{B-L} \lesssim 10^{15} \mathrm{GeV}$, corresponding to $m_{a} \gtrsim 7 \times 10^{-8} \mathrm{eV}$.

Figure 1 shows the predictions of the model in the axion-photon vs. axion-mass plane. Barring a hole in sensitivity around $m_{a} \sim 10^{-7} \mathrm{eV}$, the parameter space of the accidental $\mathrm{SO}(10)$ axion in the pre-inflationary $\mathrm{PQ}$ breaking scenario will be mostly explored in the coming decades, under the crucial assumption that the axion comprises the whole DM (otherwise the sensitivity of axion DM experiments on the axion-photon coupling is diluted as $\left.\left(\Omega_{a} / \Omega_{\mathrm{DM}}\right)^{1 / 2}\right)$.

It should be noted that although the allowed axion mass window spans over several orders of magnitude, further refinements of the present analysis will likely narrow down the axion mass range. This is due to extra constraints on the $B-L$ and PQ breaking scales originating from the requirement of reproducing SM fermion masses and mixings as well as from gauge coupling unification. However, we refrain from applying standard unification constraints to the present model, since those are affected by the replication of the $\mathrm{SO}(10)$ Higgs fields under $\mathrm{SU}(3)_{f}$, which could drastically change the running [50], depending on the pattern of $\mathrm{SU}(3)_{f}$ breaking.

\subsection{Post-inflationary PQ breaking}

If the PQ is broken after inflation or restored afterwards, one has also topological defects (axion strings and domain walls) that contribute to the axion DM relic density, on top of the usual misalignment mechanism. Such a scenario is theoretically motivated by the possibility of addressing the PQ quality problem in the present framework. In fact, using the estimate in eq. (3.8) for the induced QCD theta term and the expression of $f_{a}$ in terms 


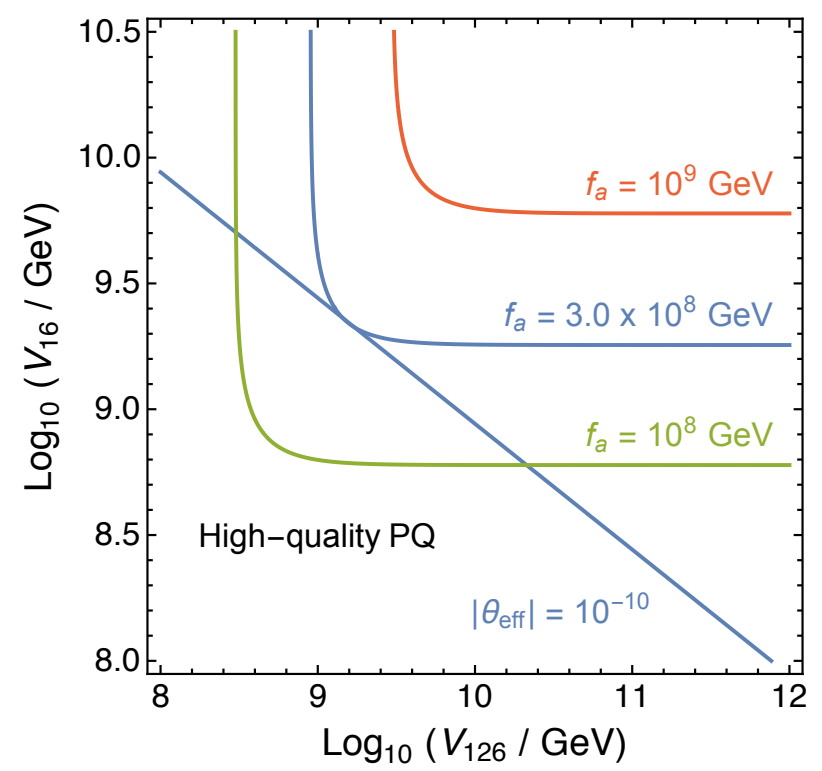

Figure 2. Parameter space of the accidental $\mathrm{SO}(10)$ axion leading to a high-quality $\mathrm{U}(1)_{\mathrm{PQ}}$. For the estimate of the induced QCD theta term we used eq. (3.8) with $|k|=1$ and $\sin \delta=1$.

of $V_{16, \overline{126}}$ (cf. eq. (4.20)), we find that in order to satisfy the nEDM bound, $\left|\theta_{\text {eff }}\right| \lesssim 10^{-10}$, one needs $f_{a} \lesssim 3.0 \times 10^{8} \mathrm{GeV}$ (correspondingly, $m_{a} \gtrsim 0.02 \mathrm{eV}$ ). The more general parameter space in terms of $V_{\overline{126}}$ and $V_{16}$ is displayed in figure 2.

In the post-inflationary PQ breaking scenario there is a main phenomenological issue given by the axion domain wall problem (recall that the model has $N_{\mathrm{DW}}=12$, see section 4.3). A small breaking of the $\mathrm{U}(1)_{\mathrm{PQ}}$ via the Planck suppressed operator in eq. (3.7) could remove the degeneracy among the vacua of the axion potential, effectively leading to the domain walls to decay before they dominate the energy density of the universe [40]. Parametrizing the PQ breaking via a bias term in the scalar potential $[41,51]$

$$
\mathcal{V}_{\text {bias }}=-2 \Xi V^{4} \cos \left(\frac{a}{V}+\delta\right)
$$

with $V=N_{\mathrm{DW}} f_{a}$, the energy density difference between two neighbouring minima, e.g. in $a=0$ and $a=2 \pi / N_{\text {DW }}$, is (for $\left.\sin \delta \approx 1\right) \Delta \mathcal{V}=\mathcal{V}_{\text {bias }}\left(2 \pi / N_{\text {DW }}\right)-\mathcal{V}_{\text {bias }}(0) \approx \Xi V^{4}(1-$ $\left.\cos \left(2 \pi / N_{\mathrm{DW}}\right)\right)$. A simple estimate of the decay time of the domain wall is obtained by equating the volume pressure $p_{V} \sim \Delta \mathcal{V}$ and the tension force $p_{T} \sim \sigma_{\mathrm{DW}} / t$ (with $\sigma_{\mathrm{DW}} \approx$ $9 m_{a} f_{a}^{2}$ denoting the wall tension $\left.[52,53]\right)$. Hence,

$$
t_{\text {decay }} \approx \frac{\sigma_{\mathrm{DW}}}{\Xi V^{4}\left(1-\cos \left(2 \pi / N_{\mathrm{DW}}\right)\right)} \approx 5 \times 10^{-5} \mathrm{~s}\left(\frac{10^{-50}}{\Xi}\right)\left(\frac{12}{N_{\mathrm{DW}}}\right)^{4}\left(\frac{m_{a}}{0.02 \mathrm{eV}}\right)^{3} .
$$

Matching the bias parameter with the potential in eq. (3.7) due to the Planck-suppressed operator $\phi_{16}^{6} \phi_{126}^{3}$, we obtain $\Xi \sim\left(V / M_{\mathrm{Pl}}\right)^{5} \approx 5 \times 10^{-48}\left(0.02 \mathrm{eV} / m_{a}\right)^{5}\left(N_{\mathrm{DW}} / 12\right)^{5}$. Hence, for $m_{a}$ in the ballpark of $0.02 \mathrm{eV}$ the domain walls safely decay before Big Bang nucleosynthesis (and also well before they start to dominate the energy density of the Universe). 
Note, however, that due to the $t_{\text {decay }} \propto m_{a}^{8}$ dependence the bound saturates fast for larger $m_{a}$ (e.g. $t_{\text {decay }} \sim 10 \mathrm{~s}$ for $\left.m_{a}=0.2 \mathrm{eV}\right)$.

For $m_{a} \gtrsim 0.02 \mathrm{eV}$, astrophysical constraints are also at play (see section 4.7 in [54] for an updated summary). In particular, limits from red giants and white dwarfs cooling on the axion-electron coupling and the SN1987A bound on the axion-nucleon couplings are in the ballpark of $m_{a} \sim 0.02 \mathrm{eV}$, depending however on electroweak vacuum parameters (cf. eqs. (A.30)-(A.31)). Their perturbativity domain will differ from those of the standard DFSZ [8,9] axion model (as it does happen in PQ-extended left-right symmetric models [55]). Hence, it is possible that astrophysical constraints could be relaxed to some extent compared to the DFSZ axion case, thus entering the region probed by IAXO in figure 1. A detailed analysis of astrophysical constraints is beyond the scopes of the present work, but we note in passing that the axion-electron coupling $g_{a e}=c_{e} m_{e} / f_{a}$ (with $c_{e}$ given in eq. (A.31)), is of the size required to explain the so-called "stellar cooling anomalies" [56] for $m_{a} \approx 0.02 \mathrm{eV}$ and $c_{e} \approx 0.1$.

Finally, it is interesting to note that recent simulations of the axion-string network suggest a much larger contribution to the total axion relic density than what traditionally thought [57, 58]. Rescaling the results of ref. [58] for a generic domain wall number $N_{\mathrm{DW}}$, $m_{a}\left(N_{\mathrm{DW}}\right)=\left(Q\left(N_{\mathrm{DW}}\right) / Q\left(N_{\mathrm{DW}}=1\right)\right)^{6 / 7} m_{a}\left(N_{\mathrm{DW}}=1\right)$ (with the function $Q$ given in eq. (36) of [58], after replacing $\xi_{\star} \log _{\star} \rightarrow N_{\mathrm{DW}}^{2} \xi_{\star} \log _{\star}$ ), one obtains a lower bound on the axion DM mass for $N_{\mathrm{DW}}=12$ of about $m_{a} \gtrsim 7.5 \mathrm{meV}$, which still neglects extra stringsdomain walls contribution after the onset of axion oscillations. Hence, the requirement of the axion comprising the whole DM could potentially be compatible also with the highquality axion mass window in figure 1.

\section{Conclusions}

Obtaining an automatic $\mathrm{U}(1)_{\mathrm{PQ}}$ in GUTs is a longstanding problem, with remarkably few successful attempts. ${ }^{11}$ Any progress in that direction would certainly make the connection between two motivated frameworks (the axion and grand unification) a more convincing one. Here, we have shown that the gauging the $\mathrm{SU}(3)_{f}$ flavour group in $\mathrm{SO}(10)$ leads to an automatic $\mathrm{U}(1)_{\mathrm{PQ}}$ if the $\mathrm{SO}(10)$ Higgs representations are properly chosen. Moreover, the $\mathrm{PQ}$ symmetry is protected also beyond the renormalizable level, thus providing a possible way to tackle the PQ quality problem. In particular, the leading contributions to the axion potential arise from $d=9$ operator which, if Planck-suppressed, imply that the axion must be relatively heavy, $m_{a} \gtrsim 0.02 \mathrm{eV}$. Disregarding instead the PQ quality problem in its standard formulation in terms of power-like effective operators, the axion can be as light as about $7 \times 10^{-8} \mathrm{eV}$, as implied by the upper bound on $f_{a}$ given by the seesaw scale. What emerges is an intermediate-scale axion, that is quite different from the usual GUT-scale

\footnotetext{
${ }^{11}$ The original work of Georgi et al. [3] based on SU(9) stands out as possibly the only successful one, that relies only on GUT dynamics. Ref. [13] proposed a SUSY $E_{6} \times \mathrm{U}(1)^{\prime}$ gauge model, where the $\mathrm{U}(1)_{\mathrm{PQ}}$ arises accidentally and it is protected against higher-dimensional operators. However, as observed in [59], the solution to the PQ quality problem is spoiled by soft SUSY breaking effects. Other approaches to the PQ quality problem based on composite dynamics such as [60], can be made compatible with GUTs.
} 
axion, $m_{a} \lesssim 10^{-9} \mathrm{eV}$, obtained by dominantly breaking the PQ symmetry at the GUT scale. In the latter case, the $\mathrm{U}(1)_{\mathrm{PQ}}$ breaking $\mathrm{SO}(10)$ representations, i.e. 45,54 or 210 , have a trivial $\mathrm{SO}(10)$ center and thus the PQ symmetry does not arise automatically. From this perspective, the GUT-scale axion and in general $\mathrm{SO}(10) \times \mathrm{U}(1)_{\mathrm{PQ}}$ models in which the PQ breaking is related to the VEV of a complex 45, 54 or 210 (even at intermediate mass scales, see e.g. [61-66]) appear to be theoretically less motivated, since it is more difficult to obtain an automatic $\mathrm{U}(1)_{\mathrm{PQ}}$ (compared to models in which the $\mathrm{U}(1)_{\mathrm{PQ}}$ is broken by $\mathrm{SO}(10)$ representations with non-trivial $B-L[15,21,27])$.

Some of the considerations above extend as well to other GUT groups. Recent nonGUT constructions based on $\mathrm{SU}(\mathcal{N})$ gauge dynamics [67, 68], showed that it is possible to protect the PQ symmetry via the $\mathbb{Z}_{\mathcal{N}}$ center of $\mathrm{SU}(\mathcal{N})$ up to operators of dimension $\mathcal{N}$. One could try to follow a similar path for GUTs. SU(5) is broken to the SM in one step, either via a 24 or a 75 , both of which have however a trivial center (in fact, all SU(5) representations containing a SM-singlet direction have also zero quintality), thus making structurally difficult to get an automatic $\mathrm{U}(1)_{\mathrm{PQ}}$ in $\mathrm{SU}(5)$ where the $\mathrm{PQ}$ symmetry is broken via a complex adjoint [69-72]. Non-minimal GUTs such as $\mathrm{SU}(6)$ or $E_{6}$ are more promising in this respect, since they feature representations with SM-singlet directions (which can achieve large VEVs compared to the electroweak scale) and at the same time transform non-trivially under their respective $\mathbb{Z}_{6}$ and $\mathbb{Z}_{3}$ centers. Pati-Salam instead looks much alike $\mathrm{SO}(10)$. For instance, the models discussed in refs. [73, 74] feature a PQ breaking representation that transforms trivially under the $\mathbb{Z}_{4}$ center of SU(4) $)_{\mathrm{PS}}$, and hence the $\mathrm{U}(1)_{\mathrm{PQ}}$ is not protected. Following a similar path as in the present work, an accidental PatiSalam axion could arise by breaking the PQ symmetry with representations that have nontrivial $B-L$ quantum numbers (e.g. a 4 and a 10) and transform non-trivially under flavour.

Finally, it is remarkable that if flavour has to play a role for the $\mathrm{U}(1)_{\mathrm{PQ}}$ to arise accidentally, three generations are indeed quite special, since for example $n_{g}=1,2,4$ would have not worked $[15,16]$. So, ironically, $n_{g}=3$ could have been responsible for making the CKM phase physical and at the same time washing out $\mathrm{CP}$ violation from strong interactions.

\section{Acknowledgments}

I thank Marco Gorghetto, Federico Mescia, Michele Redi and Giovanni Villadoro for useful discussions. This work is supported by the Marie Skłodowska-Curie Individual Fellowship grant AXIONRUSH (GA 840791) and the Deutsche Forschungsgemeinschaft under Germany's Excellence Strategy - EXC 2121 Quantum Universe - 390833306.

\section{A Low-energy axion couplings}

In this appendix we provide a derivation of low-energy $\mathrm{SO}(10)$ axion couplings to SM matter fields, ${ }^{12}$ that requires the inclusion of electroweak VEVs for the identification of

\footnotetext{
${ }^{12}$ See also ref. [65] for a similar approach to $\mathrm{SO}(10)$ axion couplings. Note that the latter paper did not consider the specific $\mathrm{SO}(10)$ model studied here.
} 


\begin{tabular}{|c|c|c|c|c|c|c|c|c|}
\hline & $\Delta^{\overline{126}}$ & $\delta^{16}$ & $H_{d}^{16}$ & $H_{u}^{10}$ & $H_{d}^{10}$ & $H_{u}^{\overline{126}}$ & $H_{d}^{\overline{126}}$ & $\Delta_{L}^{\overline{126}}$ \\
\hline $\mathrm{PQ}$ & -2 & -1 & -1 & -2 & -2 & -2 & -2 & -2 \\
$B-L$ & -2 & 1 & -1 & 0 & 0 & 0 & 0 & 2 \\
$Y$ & 0 & 0 & $-1 / 2$ & $1 / 2$ & $-1 / 2$ & $1 / 2$ & $-1 / 2$ & 1 \\
\hline
\end{tabular}

Table 2. Global (PQ) and local $(B-L$ and $Y$ ) charges of the $\mathrm{SO}(10)$ sub-multiplets hosting the physical axion field.

the physical axion field. Table 2 summarizes the global (PQ) and gauge $(B-L$ and $Y$ ) charges of the $\mathrm{SO}(10)$ sub-multiplets which host the axion as an angular component (this requires a complex scalar with a $Q=T_{L}^{3}+Y=0$ neutral component), i.e.

$$
\Phi_{i} \supset \frac{V_{i}}{\sqrt{2}} e^{i \frac{a_{i}}{V_{i}}}
$$

with $\Phi=\left\{\Delta^{\overline{126}}, \delta^{\overline{126}}, H_{d}^{16}, H_{u}^{10}, H_{d}^{10}, H_{u}^{\overline{126}}, H_{d}^{\overline{126}}, \Delta_{L}^{\overline{126}}\right\}$ spanning over the fields in table 2.

The low-energy PQ symmetry, with charge $q$, is a linear combination of the UV PQ charges and two broken Cartan generators, which can be chosen as $B-L$ and $Y$, namely

$$
q=c_{1} \mathrm{PQ}+c_{2}(B-L)+c_{3} Y
$$

It can be shown, analogously to the cases discussed in section 4, that in order to match $\mathrm{U}(1)_{\mathrm{PQ}}$ anomalies in terms of UV and IR charges, $c_{1}=1$, which we henceforth assume in the following. Given the PQ current $J_{\mu}^{\mathrm{PQ}}=\sum_{i} q_{i} V_{i} \partial_{\mu} a_{i}$ The canonical axion field is defined as

$$
a=\frac{1}{V} \sum_{i} q_{i} V_{i} a_{i}, \quad V^{2}=\sum_{i} q_{i}^{2} V_{i}^{2},
$$

so that $J_{\mu}^{\mathrm{PQ}}=V \partial_{\mu} a$ and, compatibly with the Goldstone theorem, $\left\langle 0\left|J_{\mu}^{\mathrm{PQ}}\right| a\right\rangle=i V p_{\mu}$. Under a PQ transformation $a_{i} \rightarrow a_{i}+\alpha q_{i} V_{i}$ the axion field transforms as $a \rightarrow a+\alpha V$. Inverting the orthogonal transformation in eq. (A.3), one readily obtains the projection of the angular modes on the axion field:

$$
a_{i} \rightarrow q_{i} V_{i} \frac{a}{V}
$$

To determine the $q$ charges we proceed as follows. First, we require the orthogonality between the axion current and the gauge currents $J_{B-L}=\sum_{i}(B-L)_{i} V_{i} \partial_{\mu} a_{i}$ and $J_{Y}=$ $\sum_{i} Y_{i} V_{i} \partial_{\mu} a_{i}$ (to avoid kinetic mixings of the axion field with massive gauge bosons). This yields, respectively:

$$
\begin{aligned}
-2 q_{126} V_{\frac{126}{126}}^{2}+q_{16} V_{16}^{2}-q_{H_{d}^{16}}\left(v_{16}^{d}\right)^{2} & =0, \\
-\frac{1}{2} q_{H_{d}^{16}}\left(v_{16}^{d}\right)^{2}+\frac{1}{2} q_{H_{u}^{10}}\left(v_{10}^{u}\right)^{2}-\frac{1}{2} q_{H_{d}^{10}}\left(v_{10}^{d}\right)^{2}+\frac{1}{2} q_{H_{u}^{126}}\left(v_{\overline{126}}^{u}\right)^{2}-\frac{1}{2} q_{H_{d}^{126}}\left(v_{\frac{d}{126}}\right)^{2} & =0 .
\end{aligned}
$$


Second, by decomposing the invariants with non-trivial global re-phasings in the $\mathrm{SO}(10)$ scalar potential (cf. eqs. (2.5)-(2.5)): $:^{13}$

$$
\begin{aligned}
\phi_{16}^{2} \phi_{10}^{\star} \supset H_{d}^{16} \delta^{16}\left(H_{d}^{10}\right)^{\star}, \\
\phi_{10}^{2} \phi_{\frac{126}{22}} \supset\left(H_{u}^{10}\right)^{2}\left(H_{u}^{\overline{126}}\right)^{2 \star},\left(H_{d}^{10}\right)^{2}\left(H_{d}^{\overline{126}}\right)^{2 \star},
\end{aligned}
$$

one obtains the following extra constraints on $q$ charges (recall eq. (A.1) and eq. (A.4)):

$$
\begin{array}{r}
q_{H_{d}^{16}}+q_{16}-q_{H_{d}^{10}}=0, \\
q_{H_{u}^{10}}-q_{H_{u}^{126}}=q_{H_{d}^{10}}-q_{H_{d}^{126}}=0,
\end{array}
$$

from which we see that the (electroweak) $q$ charges of $\phi_{10}$ and $\phi_{\overline{126}}$ are aligned.

To close the system of linear equations, in order to extract the $q$ charges, it is necessary to include also the matching between UV and IR PQ charges in eq. (A.2) for all the scalar fields. Here, we present the result in the simplifying limit $v_{H_{d}^{16}} \rightarrow 0$ (although for the final expressions of axion couplings we will keep $v_{H_{d}^{16}} \neq 0$ ), that is

$$
\begin{aligned}
c_{2} & =\frac{V_{16}^{2}-4 V_{126}^{2}}{V_{16}^{2}+4 V_{126}^{2}}, \\
c_{3} & =4 \frac{v_{u}^{2}-v_{d}^{2}}{v^{2}}, \\
q_{\overline{126}} & =-\frac{4 V_{16}^{2}}{V_{16}^{2}+4 V_{\overline{126}}^{2}}, \\
q_{16} & =-\frac{8 V_{126}^{2}}{V_{16}^{2}+4 V_{126}^{2}}, \\
q_{H_{d}^{16}} & =\frac{8 V_{126}^{2}\left(v_{d}^{2}-v_{u}^{2}\right)-4 V_{16}^{2} v_{u}^{2}}{\left(V_{16}^{2}+4 V_{126}^{2}\right) v^{2}}, \\
q_{H_{u}^{10}} & =q_{H_{u}^{126}}=-4 \frac{v_{d}^{2}}{v^{2}}, \\
q_{H_{d}^{10}} & =q_{H_{d}^{126}}=-4 \frac{v_{u}^{2}}{v^{2}}, \\
V^{2} & =16\left(\frac{V_{16}^{2} V_{126}^{2}}{V_{16}^{2}+4 V_{\overline{126}}^{2}}+\frac{v_{u}^{2} v_{d}^{2}}{v^{2}}\right) .
\end{aligned}
$$

where we defined $v_{u}^{2}=v_{H_{u}^{10}}^{2}+v_{H_{u}^{\overline{126}}}^{2}, v_{d}^{2}=v_{H_{d}^{10}}^{2}+v_{H_{d}^{\overline{126}}}^{2}$ and $v^{2}=v_{u}^{2}+v_{d}^{2}$.

To compute low-energy axion couplings to SM charged fermions, we decompose $\psi_{16} \psi_{16} \phi_{10} \supset q u^{c} H_{u}^{10}+q d^{c} H_{d}^{10}+\ldots$. The axion is removed from the Yukawa interaction via a family universal axion-dependent transformation (in Weyl notation and suppressing

\footnotetext{
${ }^{13}$ In the following, we set to zero the $\mathrm{SU}(2)_{L}$ triplet $\mathrm{VEV}, v_{\Delta_{L}^{126}}$. Keeping the latter, axion couplings to SM charged fermions receive safely negligible corrections of the order of $v_{\Delta_{L}^{126}}^{2} / v^{2} \ll 1$ (with $v$ denoting the electroweak scale) [75].
} 
generation indices)

$$
\begin{array}{ll}
u \rightarrow e^{-i q_{H_{u}^{10}} \frac{a}{2 V}} u, & u^{c} \rightarrow e^{-i q_{H_{u}^{10}} \frac{a}{2 V}} u^{c}, \\
d \rightarrow e^{-i q_{H_{d}^{10} \frac{a}{2 V}}} d, & d^{c} \rightarrow e^{-i q_{H_{d}^{10} \frac{a}{2 V}}^{c}} d^{c},
\end{array}
$$

(equivalently, in Dirac notation, $u \rightarrow e^{i q_{H_{u}^{10}} \gamma_{5} \frac{a}{2 V}} u$, etc.), and analogously for charged leptons. The same rotation also removes the axion from the other Yukawa interaction, $\psi_{16} \psi_{16} \phi_{\overline{126}}^{\star}$, due to the alignment of the (electroweak) PQ charges of $\phi_{10}$ and $\phi_{\overline{126}}$.

The SM chiral fermion transformation above generate the anomalous terms

$$
\delta \mathscr{L}=\frac{\alpha_{s} N}{4 \pi} \frac{a}{V} G \tilde{G}+\frac{\alpha E}{4 \pi} \frac{a}{V} F \tilde{F},
$$

with the anomaly factors

$$
\begin{aligned}
& N=-n_{g} T(3)\left(2 \frac{q_{H_{u}^{10}}}{2}+2 \frac{q_{H_{d}^{10}}}{2}\right)=6, \\
& E=-n_{g}\left(q_{H_{u}^{10}} 3(2 / 3)^{2}+q_{H_{d}^{10}} 3(-1 / 3)^{2}+q_{H_{d}^{10}}(-1)^{2}\right)=16,
\end{aligned}
$$

using $n_{g}=3, T(3)=1 / 2$, etc. So, in particular, upon choosing the standard normalization of the axion-gluon coupling in terms of the Lagrangian term

$$
\frac{\alpha_{s}}{8 \pi} \frac{a}{f_{a}} G \tilde{G},
$$

the axion decay constant reads (using eq. (A.18) with the sign fixed as in eq. (4.20) for the case of no electroweak VEVs)

$$
f_{a}=\frac{V}{2 N}=-\frac{1}{3} \sqrt{\frac{V_{16}^{2} V_{126}^{2}}{V_{16}^{2}+4 V_{\overline{126}}^{2}}+\frac{v_{u}^{2} v_{d}^{2}}{v^{2}}},
$$

while $E / N=8 / 3$, which enters the axion-photon coupling [76]

$$
C_{a \gamma}=E / N-1.92(4),
$$

defined in terms of the Lagrangian term $\mathscr{L} \supset \frac{\alpha}{8 \pi f_{a}} C_{a \gamma} a F \tilde{F}$.

On the other hand, the variation of the fermion kinetic terms yields (in Dirac notation)

$$
\begin{aligned}
\delta(\bar{u} i \not \partial u) & =-q_{H_{u}^{10}} \frac{\partial_{\mu} a}{2 V} \bar{u} \gamma^{\mu} \gamma_{5} u, \\
\delta(\bar{d} i \not \partial d) & =-q_{H_{d}^{10}} \frac{\partial_{\mu} a}{2 V} \bar{d} \gamma^{\mu} \gamma_{5} d, \\
\delta(\bar{e} i \not \partial e) & =-q_{H_{d}^{10}} \frac{\partial_{\mu} a}{2 V} \bar{e} \gamma^{\mu} \gamma_{5} e .
\end{aligned}
$$

Hence, defining the axion coupling to SM fermions via $\mathscr{L} \supset \frac{\partial_{\mu} a}{2 f_{a}} c_{f} \bar{f} \gamma_{\mu} \gamma_{5} f$, and replacing $f_{a}=V /(2 N)$, we find the (family universal) couplings

$$
\begin{aligned}
& c_{u}=-\frac{q_{H_{u}^{10}}}{2 N}=\frac{1}{3} \frac{V_{16}^{2}\left(v_{d}^{2}+v_{H_{d}^{16}}^{2}\right)+2 V_{126}^{2}\left(2 v_{d}^{2}+v_{H_{d}^{16}}^{2}\right)+v_{d}^{2} v_{H_{d}^{16}}^{2}}{\left(V_{16}^{2}+4 V_{126}^{2}\right)\left(v^{2}+v_{H_{d}^{16}}^{2}\right)+v^{2} v_{H_{d}^{16}}^{2}} \approx \frac{v_{d}^{2}}{3 v^{2}}, \\
& c_{d}=c_{e}=-\frac{q_{H_{u}^{10}}}{2 N}=\frac{1}{3} \frac{V_{16}^{2} v_{u}^{2}+2 V_{126}^{2}\left(2 v_{u}^{2}+v_{H_{d}^{16}}^{2}\right)+v_{u}^{2} v_{H_{d}^{16}}^{2}}{\left(V_{16}^{2}+4 V_{126}^{2}\right)\left(v^{2}+v_{H_{d}^{16}}^{2}\right)+v^{2} v_{H_{d}^{16}}^{2}} \approx \frac{v_{u}^{2}}{3 v^{2}},
\end{aligned}
$$

where in the last step we provided simplified expressions in the $v_{H_{d}^{16}} \rightarrow 0$ limit. 
Open Access. This article is distributed under the terms of the Creative Commons Attribution License (CC-BY 4.0), which permits any use, distribution and reproduction in any medium, provided the original author(s) and source are credited.

\section{References}

[1] R.D. Peccei and H.R. Quinn, CP Conservation in the Presence of Instantons, Phys. Rev. Lett. 38 (1977) 1440 [INSPIRE].

[2] R.D. Peccei and H.R. Quinn, Constraints Imposed by CP Conservation in the Presence of Instantons, Phys. Rev. D 16 (1977) 1791 [INSPIRE].

[3] H.M. Georgi, L.J. Hall and M.B. Wise, Grand Unified Models With an Automatic \{Peccei-Quinn\} Symmetry, Nucl. Phys. B 192 (1981) 409 [inSPIRE].

[4] S. Weinberg, A New Light Boson?, Phys. Rev. Lett. 40 (1978) 223 [InSPIRE].

[5] F. Wilczek, Problem of Strong $P$ and T Invariance in the Presence of Instantons, Phys. Rev. Lett. 40 (1978) 279 [INSPIRE].

[6] J.E. Kim, Weak Interaction Singlet and Strong CP Invariance, Phys. Rev. Lett. 43 (1979) 103 [INSPIRE].

[7] M.A. Shifman, A.I. Vainshtein and V.I. Zakharov, Can Confinement Ensure Natural CP Invariance of Strong Interactions?, Nucl. Phys. B 166 (1980) 493 [InSPIRE].

[8] A.R. Zhitnitsky, On Possible Suppression of the Axion Hadron Interactions (in Russian), Sov. J. Nucl. Phys. 31 (1980) 260 [inSPIRE].

[9] M. Dine, W. Fischler and M. Srednicki, A Simple Solution to the Strong CP Problem with a Harmless Axion, Phys. Lett. B 104 (1981) 199 [INSPIRE].

[10] M. Dine and N. Seiberg, String Theory and the Strong $\{C P\}$ Problem, Nucl. Phys. B 273 (1986) 109 [INSPIRE].

[11] S.M. Barr and D. Seckel, Planck scale corrections to axion models, Phys. Rev. D 46 (1992) 539 [INSPIRE].

[12] M. Kamionkowski and J. March-Russell, Planck scale physics and the Peccei-Quinn mechanism, Phys. Lett. B 282 (1992) 137 [hep-th/9202003] [INSPIRE].

[13] R. Holman, S.D.H. Hsu, T.W. Kephart, E.W. Kolb, R. Watkins and L.M. Widrow, Solutions to the strong CP problem in a world with gravity, Phys. Lett. B 282 (1992) 132 [hep-ph/9203206] [INSPIRE].

[14] S. Ghigna, M. Lusignoli and M. Roncadelli, Instability of the invisible axion, Phys. Lett. B 283 (1992) 278 [INSPIRE].

[15] D. Chang and G. Senjanović, On Axion and Familons, Phys. Lett. B 188 (1987) 231 [INSPIRE].

[16] D. Chang, P.B. Pal and G. Senjanović, Axions From Chiral Family Symmetry, Phys. Lett. B 153 (1985) 407 [INSPIRE].

[17] P.B. Pal, The Strong CP question in $\mathrm{SU}(3)(C) \times \mathrm{SU}(3)(L) \times \mathrm{U}(1)(N)$ models, Phys. Rev. $D$ 52 (1995) 1659 [hep-ph/9411406] [INSPIRE].

[18] K.S. Babu and R.N. Mohapatra, Predictive neutrino spectrum in minimal $\mathrm{SO}(10)$ grand unification, Phys. Rev. Lett. 70 (1993) 2845 [hep-ph/9209215] [INSPIRE]. 
[19] K. Matsuda, Y. Koide and T. Fukuyama, Can the $\mathrm{SO}(10)$ model with two Higgs doublets reproduce the observed fermion masses?, Phys. Rev. D 64 (2001) 053015 [hep-ph/0010026] [INSPIRE].

[20] K. Matsuda, Y. Koide, T. Fukuyama and H. Nishiura, How far can the $\mathrm{SO}(10)$ two Higgs model describe the observed neutrino masses and mixings?, Phys. Rev. D 65 (2002) 033008 [Erratum ibid. 65 (2002) 079904] [hep-ph/0108202] [INSPIRE].

[21] B. Bajc, A. Melfo, G. Senjanović and F. Vissani, Yukawa sector in non-supersymmetric renormalizable SO(10), Phys. Rev. D 73 (2006) 055001 [hep-ph/0510139] [InSPIRE].

[22] L. Di Luzio, Aspects of symmetry breaking in Grand Unified Theories, Ph.D. thesis, SISSA, Trieste, 2011. arXiv:1110.3210 [INSPIRE].

[23] S. Bertolini, L. Di Luzio and M. Malinsky, On the vacuum of the minimal nonsupersymmetric SO(10) unification, Phys. Rev. D 81 (2010) 035015 [arXiv:0912.1796] [InSPIRE].

[24] S. Bertolini, L. Di Luzio and M. Malinsky, Seesaw Scale in the Minimal Renormalizable SO(10) Grand Unification, Phys. Rev. D 85 (2012) 095014 [arXiv:1202.0807] [InSPIRE].

[25] S. Bertolini, L. Di Luzio and M. Malinsky, Light color octet scalars in the minimal SO(10) grand unification, Phys. Rev. D 87 (2013) 085020 [arXiv:1302.3401] [INSPIRE].

[26] L. Gráf, M. Malinský, T. Mede and V. Susič, One-loop pseudo-Goldstone masses in the minimal SO(10) Higgs model, Phys. Rev. D 95 (2017) 075007 [arXiv:1611.01021] [InSPIRE].

[27] R.N. Mohapatra and G. Senjanović, The Superlight Axion and Neutrino Masses, Z. Phys. C 17 (1983) 53 [inSPIRE].

[28] R. Slansky, Group Theory for Unified Model Building, Phys. Rept. 79 (1981) 1 [INSPIRE].

[29] L.F. Abbott and M.B. Wise, Wormholes and Global Symmetries, Nucl. Phys. B 325 (1989) 687 [INSPIRE].

[30] S.R. Coleman and K.-M. Lee, Wormholes made without massless matter fields, Nucl. Phys. B 329 (1990) 387 [INSPIRE].

[31] R. Kallosh, A.D. Linde, D.A. Linde and L. Susskind, Gravity and global symmetries, Phys. Rev. D 52 (1995) 912 [hep-th/9502069] [InSPIRE].

[32] R. Alonso and A. Urbano, Wormholes and masses for Goldstone bosons, JHEP 02 (2019) 136 [arXiv: 1706.07415] [INSPIRE].

[33] A. Hebecker, T. Mikhail and P. Soler, Euclidean wormholes, baby universes, and their impact on particle physics and cosmology, Front. Astron. Space Sci. 5 (2018) 35 [arXiv:1807.00824] [INSPIRE].

[34] D. Chang, R.N. Mohapatra, J. Gipson, R.E. Marshak and M.K. Parida, Experimental Tests of New SO(10) Grand Unification, Phys. Rev. D 31 (1985) 1718 [InSPIRE].

[35] N.G. Deshpande, E. Keith and P.B. Pal, Implications of LEP results for $\mathrm{SO}(10)$ grand unification with two intermediate stages, Phys. Rev. D 47 (1993) 2892 [hep-ph/9211232] [INSPIRE].

[36] S. Bertolini, L. Di Luzio and M. Malinsky, Intermediate mass scales in the non-supersymmetric $\mathrm{SO}(10)$ grand unification: A Reappraisal, Phys. Rev. D 80 (2009) 015013 [arXiv: 0903.4049] [INSPIRE].

[37] G. Lazarides and Q. Shafi, Axion Models with No Domain Wall Problem, Phys. Lett. B 115 (1982) 21 [INSPIRE]. 
[38] S.M. Barr, D.B. Reiss and A. Zee, Families, the Invisible Axion, and Domain Walls, Phys. Lett. B 116 (1982) 227 [INSPIRE].

[39] G. Lazarides and Q. Shafi, Axion Model with Intermediate Scale Fermionic Dark Matter, Phys. Lett. B 807 (2020) 135603 [arXiv:2004.11560] [INSPIRE].

[40] P. Sikivie, Of Axions, Domain Walls and the Early Universe, Phys. Rev. Lett. 48 (1982) 1156 [INSPIRE].

[41] M. Kawasaki, K. Saikawa and T. Sekiguchi, Axion dark matter from topological defects, Phys. Rev. D 91 (2015) 065014 [arXiv: 1412.0789] [INSPIRE].

[42] Z. Berezhiani and F. Nesti, Supersymmetric $\mathrm{SO}(10)$ for fermion masses and mixings: Rank-1 structures of flavor, JHEP 03 (2006) 041 [hep-ph/0510011] [INSPIRE].

[43] M. Joyce and N. Turok, Family symmetry, fermion mass matrices and cosmic texture, Nucl. Phys. B 416 (1994) 389 [hep-ph/9301287] [INSPIRE].

[44] C. O'Hare, cajohare/axionlimits: Axionlimits, https://doi.org/10.5281/zenodo.3932430.

[45] S. Borsányi et al., Calculation of the axion mass based on high-temperature lattice quantum chromodynamics, Nature 539 (2016) 69 [arXiv:1606.07494] [INSPIRE].

[46] Particle Data Group collaboration, Review of Particle Physics, Phys. Rev. D 98 (2018) 030001 [INSPIRE].

[47] O. Wantz and E.P.S. Shellard, Axion Cosmology Revisited, Phys. Rev. D 82 (2010) 123508 [arXiv:0910.1066] [INSPIRE].

[48] E. Witten, Neutrino Masses in the Minimal O(10) Theory, Phys. Lett. B 91 (1980) 81 [INSPIRE].

[49] B. Bajc and G. Senjanović, Radiative seesaw: A Case for split supersymmetry, Phys. Lett. B 610 (2005) 80 [hep-ph/0411193] [INSPIRE].

[50] A. Banerjee, G. Bhattacharyya and P.B. Pal, $\mathrm{SO}(10)$ unification with horizontal symmetry, Phys. Rev. D 102 (2020) 015018 [arXiv:2001.08762] [INSPIRE].

[51] K. Harigaya and M. Kawasaki, QCD axion dark matter from long-lived domain walls during matter domination, Phys. Lett. B $\mathbf{7 8 2}$ (2018) 1 [arXiv:1802.00579] [INSPIRE].

[52] M.C. Huang and P. Sikivie, The Structure of Axionic Domain Walls, Phys. Rev. D 32 (1985) 1560 [INSPIRE].

[53] T. Hiramatsu, M. Kawasaki, K. Saikawa and T. Sekiguchi, Axion cosmology with long-lived domain walls, JCAP 01 (2013) 001 [arXiv:1207.3166] [INSPIRE].

[54] L. Di Luzio, M. Giannotti, E. Nardi and L. Visinelli, The landscape of QCD axion models, Phys. Rept. 870 (2020) 1 [arXiv: 2003.01100] [INSPIRE].

[55] S. Bertolini, L. Di Luzio and F. Nesti, Axion-mediated forces, CP-violation and left-right interactions, arXiv:2006.12508 [INSPIRE].

[56] M. Giannotti, I.G. Irastorza, J. Redondo, A. Ringwald and K. Saikawa, Stellar Recipes for Axion Hunters, JCAP 10 (2017) 010 [arXiv:1708.02111] [INSPIRE].

[57] M. Gorghetto, E. Hardy and G. Villadoro, Axions from Strings: the Attractive Solution, JHEP 07 (2018) 151 [arXiv: 1806. 04677] [INSPIRE].

[58] M. Gorghetto, E. Hardy and G. Villadoro, More Axions from Strings, arXiv: 2007.04990 [INSPIRE]. 
[59] B.A. Dobrescu, The Strong CP problem versus Planck scale physics, Phys. Rev. D 55 (1997) 5826 [hep-ph/9609221] [INSPIRE].

[60] M. Redi and R. Sato, Composite Accidental Axions, JHEP 05 (2016) 104 [arXiv: 1602.05427] [INSPIRE].

[61] D.B. Reiss, Invisible axion at an intermediate symmetry breaking scale, Phys. Lett. B 109 (1982) 365 [INSPIRE].

[62] G. Lazarides, $\mathrm{SO}(10)$ and the Invisible Axion, Phys. Rev. D 25 (1982) 2425 [InSPIRE].

[63] R. Holman, G. Lazarides and Q. Shafi, Axions and the Dark Matter of the Universe, Phys. Rev. D 27 (1983) 995 [INSPIRE].

[64] G. Altarelli and D. Meloni, A non supersymmetric $\mathrm{SO}(10)$ grand unified model for all the physics below $M_{G U T}, J H E P 08$ (2013) 021 [arXiv: 1305.1001] [INSPIRE].

[65] A. Ernst, A. Ringwald and C. Tamarit, Axion Predictions in $\mathrm{SO}(10) \times \mathrm{U}(1)_{\mathrm{PQ}}$ Models, JHEP 02 (2018) 103 [arXiv: 1801.04906] [INSPIRE].

[66] S.M. Boucenna, T. Ohlsson and M. Pernow, A minimal non-supersymmetric $\mathrm{SO}(10)$ model with Peccei-Quinn symmetry, Phys. Lett. B 792 (2019) 251 [Erratum ibid. 797 (2019) 134902] [arXiv: 1812 .10548] [INSPIRE].

[67] L. Di Luzio, E. Nardi and L. Ubaldi, Accidental Peccei-Quinn symmetry protected to arbitrary order, Phys. Rev. Lett. 119 (2017) 011801 [arXiv: 1704.01122] [INSPIRE].

[68] M. Ardu, L. Di Luzio, G. Landini, A. Strumia, D. Teresi and J.-W. Wang, Axion quality from the (anti)symmetric of $\mathrm{SU}(N)$, arXiv:2007.12663 [INSPIRE].

[69] M.B. Wise, H. Georgi and S.L. Glashow, SU(5) and the Invisible Axion, Phys. Rev. Lett. 47 (1981) 402 [INSPIRE].

[70] L. Di Luzio, A. Ringwald and C. Tamarit, Axion mass prediction from minimal grand unification, Phys. Rev. D 98 (2018) 095011 [arXiv: 1807.09769] [INSPIRE].

[71] P. Fileviez Pérez, C. Murgui and A.D. Plascencia, Axion Dark Matter, Proton Decay and Unification, JHEP 01 (2020) 091 [arXiv:1911.05738] [INSPIRE].

[72] P. Fileviez Pérez, C. Murgui and A.D. Plascencia, The QCD Axion and Unification, JHEP 11 (2019) 093 [arXiv: 1908.01772] [INSPIRE].

[73] L. Di Luzio, Pati-Salam Axion, JHEP 07 (2020) 071 [arXiv : 2005. 00012] [INSPIRE].

[74] S. Saad, Fermion Masses and Mixings, Leptogenesis and Baryon Number Violation in Pati-Salam Model, Nucl. Phys. B 943 (2019) 114630 [arXiv:1712.04880] [inSPIRE].

[75] S. Bertolini, L. Di Luzio and F. Nesti, Axion properties in Left-Right symmetric models, in preparation (2020).

[76] G. Grilli di Cortona, E. Hardy, J. Pardo Vega and G. Villadoro, The QCD axion, precisely, JHEP 01 (2016) 034 [arXiv: 1511.02867] [INSPIRE]. 